\title{
A variational model of irrigation patterns
}

\author{
F. MADDALEnA ${ }^{\dagger}$ AND S. SOlimini ${ }^{\dagger}$ \\ Dipartimento di Matematica, Politecnico di Bari, \\ Via Orabona, 4, 70125 Bari, Italy \\ AND \\ J.-M. MOREL ${ }^{\S}$ \\ CMLA, École Normale Supérieure de Cachan, \\ 61 av. du Président Wilson, 94235 Cachan Cédex, France
}

[Received 30 July 2002 and in revised form 20 July 2003]

\begin{abstract}
Irrigation and draining systems, plants and trees together with their root systems, lungs and cardiovascular systems have a common morphology which seems to derive from topological constraints together with energy saving requirements. All of these systems look like spatial trees and succeed in spreading out a fluid from a source onto a volume. The associated morphology is a tree of bifurcating vessels. Their intuitive explanation is that transport energy is saved by using broad vessels as long as possible rather than thin spread out vessels. In this paper, we define a general formalism dealing with irrigation patterns. Related to martingale theory, this formalism permits one to define irrigation trees and their vessels, to give a generic form to their energy, and to show compactness for the irrigation patterns with bounded energy as well as a lower semicontinuity result for the cost functional. As a consequence, we show that a variety of source to volume irrigation problems are well posed.
\end{abstract}

\section{Introduction}

The function of many natural flow systems is to connect by a fluid flow a finite size volume to a source. This happens with drainage networks, actual plants and trees, root systems, bronchial systems, cardiovascular systems [19]. Typically, the network system is designed according to the following principles: (i) The network supplies an entire volume of an organism and a space filling hierarchical branching pattern is required; (ii) the biological networks have evolved to minimize energy dissipation. Sometimes two more principles are added, namely (iii) the size of the final branches of the network is a size-invariant unit, and (iv) the equality of flow supply through the network system [25], [26], [27], [3]. In the case of trees and plants, the energy criterion must be related to the mechanical stability of the trunk and branches in response to wind and gravity. In the case of irrigation networks, the energy criterion aims at a reduction of the overall resistance of the system.

To be able to derive quantitative properties from this set of principles, a basic assumption is usually made, namely that the network has a branched tree structure made at each scale of tubes of

\footnotetext{
${ }^{\dagger}$ Email: maddalen@dm.uniba.it

Email: solimini@dm.uniba.it

${ }^{\S}$ Email: morel@cmla.ens-cachan.fr
} 
a certain uniform length, radius and with a given branching number. In other terms, the irrigation system is a fully homogeneous tree in scales, sizes and shapes. Then, under these somewhat ad hoc assumptions, the above principles have been shown by the just mentioned authors to imply that the network has a fractal-like structure with self-similar properties. The irrigation network is then characterized by how the branching ratios, and the ratios of radii and lengths of the tubes change through the network. The above principles permit one to conclude that the branching ratios are constant, say $n$, and the radii and length ratios scale as powers of $n$. This heuristic reasoning ends up with a structure described as a self-similar fractal [25], [26], [27]. Let us mention that in geomorphology, an early study of the fractal-like behavior of natural drainage networks was started by R. E. Horton [14], A. N. Strahler [20], and generalized by E. Tokunaga [22].

The above treatment has some weak points, namely, the assumption of the existence of a network structure doing the job, the assumption that the network is a tree with countable branches and the very strong homogeneity assumption involved in heuristic calculations. These properties should be deduced from first principles, a basic variational principle related to the cost of irrigation should be at the basis of both facts, as requested in [25]. Authors in [27] acknowledge that In spite of the very large number of numerical and empirical studies, no general theory based on fundamental laws has yet been developed for (...) fractal behavior (...). It is our purpose here to discuss the first mentioned assumption, namely the proper existence of an irrigation tree irrigating a volume and with minimal resistance. This paper is not, however, the first mathematical attempt and we know of two other works addressing the existence problem, namely [5] and [29].

Before describing those works, let us remark that, in spite of the many and diverse modelling aspects involved in the formalization of the irrigation trees, it seems sound in the first mathematical inquiries to adopt the simplest variational formalization coping with the essential features of the problem. This same Occam's razor principle is adopted in the just mentioned mathematical references.

In [5], the problem of finding a maximal irrigated volume with minimal cost is addressed. Let $\Omega$ be a fixed open domain. A point source $S \in \Omega$ is fixed. We say that a compact $K \subset \Omega$ is irrigable if the complementary set $U=\Omega \backslash K$ is connected and contains $S$. $U$ is called the irrigation network. The authors fix an "accessibility profile", namely a function $f: \mathbb{R}_{+} \rightarrow \mathbb{R}_{+}$, increasing and such that $f(0)=0$. A point $x \in K$ is said $f$-irrigable if there is a path $x(s)$ such that $x(0)=x$, $x(L)=S$, and for every $s \in[0, L], B(x(s), f(s)) \subset U$, where $B(x, r)$ denotes the ball with center $x$ and radius $r$. In other terms, there is a thick path inside $U$ leading to $x$. This path becomes thinner when approaching the irrigated point, but with a thinning rate uniformly bounded from below. The authors show first that if $f$ slightly superlinear at 0 (e.g. $f(s)=s^{\alpha}, 0<\alpha<1$ ) then the problem of irrigating a maximal positive volume is well posed. Namely: there exists $K$ with maximal volume among all $f$-irrigable sets. Next, a cost functional is associated with each accessibility path to $x$. This cost functional is assumed to be lower semicontinuous with respect to the uniform convergence of paths. $K$ being fixed, one can therefore associate with each $x \in K$ a minimal accessibility cost $c_{K}(x)$. The irrigation cost of $K$ is then defined as $c(K)=\int_{K} c_{K}(x) \mathrm{d} x$. Then the existence of a maximal irrigated volume $K$ with minimal cost $c(K)$ is proved. To the best of our knowledge, this paper is the only one addressing the existence of thick embedded irrigation networks. Its weak point is the somewhat floppy expression of the cost functional.

Qinglan Xia's paper [29] was communicated to us while we were in the course of revising the present paper. Although the mathematical treatment proposed by this author and ours are very different, the considered energy functional is exactly the same and the addressed end problem is 
also essentially the same. Xia treats the irrigation problem as a transport problem from a fixed probability measure $\mu^{+}$to a fixed probability measure $\mu^{-}$. He does not consider as in [5] a volume representation of the network but treats it roughly as an embedded graph with a countable number of vertices and satisfying the Kirchhoff law. The network is then elegantly represented as a onedimensional flat current $G$ with non-integer multiplicity satisfying

$$
\partial G=\mu^{+}-\mu^{-}
$$

(in the earlier version of this paper), or, equivalently, as a vector measure supported by a countable graph (in the later version). The multiplicity of each edge of the graph represents the fluid flow along the vertex and the condition $\partial G=\mu^{+}-\mu^{-}$implies that the Kirchhoff law is satisfied at each vertex of the graph. When such graphs are finite, Xia considers the same energy we shall consider, namely

$$
E^{\alpha}(G)=\sum_{e \text { edge of } G} w(e)^{\alpha} \text { length }(e),
$$

where $0<\alpha<1$. (When the graph is infinite, he uses a relaxed version of the same functional.) This energy deserves some explanation. Assume that a tube or a branch $e$ bifurcates into two smaller edges $e_{1}$ et $e_{2}$. Then by the Kirchhoff law, $w(e)=w\left(e_{1}\right)+w\left(e_{2}\right)$. It is immediately seen that if $\alpha=1$, there is no energy loss in this bifurcation, while if $\alpha<1$, it would be more advantageous from the energy saving viewpoint to avoid a bifurcation. In fluid mechanics, this amounts to stating a qualitative Poiseuille law, according to which the resistance of a tube increases when it gets thinner. In the case of trees, the same energy criterion translates the fact that mechanical strength is increased by avoiding branching. In the case of the transportation problem addressed in [29], the interpretation reads as follows: In shipping two items from nearby cities to the same far away city, it may be less expensive to first bring them into a common location and put them on a single truck for most of the transport. In this case, a " $Y$ shaped" path is preferable to a " $V$ shaped" path". The choice of the power function $w^{\alpha}$ with $0<\alpha<1$ is just a way to express the above requirement by an example. Clearly, any function concave near 0 would do the same job for the mathematical discussion. In the following, we shall refer to Xia's approach as the "Eulerian" approach.

Let us return to our aims here. We shall consider a single source $\mu^{+}=\delta_{S}$, a Dirac mass, and any irrigated measure $\mu^{-}$. The mathematical approach developed here is actually different from the one proposed in Xia's paper. An irrigation system is not defined as an embedded graph, but as an (usually uncountable) set of paths or "fibers" starting from the source and arriving at every point of the support of the irrigated measure. Our initial search space for a solution will therefore be larger, since it allows a priori spreading trees where each fiber of a set with positive measure could go its way without following a branch. (This is an abstraction of what happens with grass, where no trunk or branch is formed and the paths, coinciding with the grass leaves, are numerous but thin and straight.) We shall refer to this approach as "Lagrangian", as opposed to the Eulerian one. Let us give some details.

Our formalism considers paths starting from the source and representing, according to the different interpretations, either the trajectory in $\mathbb{R}^{N}$ of a fluid particle, or a fiber of a tree. We shall call these infinitely many paths "fibers" and denote them by $\chi(p, t) \in \mathbb{R}^{N}$, where $t$ is time (or length along the fiber) and $p$ denotes a particle, belonging to an abstract probability space $\Omega$. We shall introduce a stopping time $\sigma_{\chi}(p)$ for each fiber, namely the first time it stops for ever. This will allow us to define the irrigation measure, as a density measure of the fibers stopping in any given volume. 
In Section 1, we define what we call a set of fibers, namely, a set of trajectories $\chi_{p}(t)$ starting all from a source point $\chi_{p}(0)=S$ and indexed by a probability space $p \in \Omega$. We then define $\chi^{-}$ vessels, or branches, as equivalence classes under the equivalence relation $p \simeq q$ if $\chi_{p}(s)$ and $\chi_{q}(s)$ coincide up to time $t$. We also define " $\chi$-flows at time $t$ " as $\chi$-vessels which have not yet stopped. Next, we address all measurability questions raised by this formalism, related in particular to the stopping or absorption time $\sigma_{\chi}(p)$ and the measurability of branches or $\chi$-vessels. We define what we call afterwards "non-spread flow", namely sets of fibers which remain all in branches ( $\chi$-flows) with positive measure or stop. All the notations and definitions are summarized in the index at the end of this paper.

In Section 2, we define our cost functional as an integral over the set of $\chi$-flows. This functional is equivalent to the above mentioned energy (0.1), defined in [29]. We make precise the notion of pointwise convergence for sets of fibers.

In Section 3 the irrigation measure is defined and its weak continuity with respect to pointwise convergence of the sets of fibers is proved. Section 6 shows the lower semicontinuity of the cost functional under pointwise convergence.

Section 4 proves a technically useful result, namely that a non-spread set of fibers can be reconstructed from the knowledge of the $\chi$-flows for a dense subset of values of $t$.

Section 5 addresses a structural invariance of the problem, namely the invariance of the irrigation measure and cost functional under any measure preserving bijection of the $\chi$-flows.

Section 7] explains why we cannot have a compactness result for the set of fibers. This is easily explained: we can exchange wildly the fibers by measure preserving maps in the minimizing process, thus obstructing the convergence of fibers while the $\chi$-flows intuitively converge. Thus, Sections 7 and 8 reduce the set of fibers to what we call "histograms", which correspond roughly to equivalence classes under measure preserving rearrangements of the fibers. Compactness properties of histograms are proved (they simply derive from the Ascoli-Arzelà theorem).

The conclusive Section 9 shows our main existence result, of a set of fibers solving the irrigation problem of a source to a given measure, as well as variants involving several measures, sources and interactions of trees like in the bronchial-cardiovascular system.

At this point, a comparison between the Lagrangian and Eulerian formalisms and results would be premature, but the impatient reader can go directly to Subsection 9.1. Both solutions turn out to be equivalent as far as existence is concerned, and complementary as for the structure they give to the solution. All necessary tools turn out to be available in the union of both papers to prove this equivalence, and we give a (sketchy) proof in the above-mentioned subsection.

Needless to say, the results given here and by the above-mentioned authors open more questions than they solve. In particular, existence results open the way to the structural questions (homogeneity of the irrigation tree, scaling laws...) for which, for the time being, only heuristic results seem at hand.

\section{Absorption time, flows and irrigation patterns}

If $X$ is a subset of $\mathbb{R}^{N}$, we shall denote by $\mathcal{H}^{\alpha}(X)$ the outer Hausdorff measure of dimension $\alpha$ of $X$ and by $|X|$ its outer Lebesgue measure. If $u$ is any real function defined on a subset $\Omega \subset \mathbb{R}^{N}$, we shall use the notation osc $u=\sup u-\inf u,\|u\|_{\infty}=\sup |u(x)|$.

Let $(\Omega,|\cdot|)$ be a probability space which we interpret as the reference configuration of a fluid incompressible material body. (We can also interpret it as the trunk section of a tree, this trunk being thought of as a set of fibers which can bifurcate into branches.) A set of fibers of $\Omega$ with source point 
$S \in \mathbb{R}^{N}$ is a mapping

$$
\chi: \Omega \times \mathbb{R}_{+} \rightarrow \mathbb{R}^{N}
$$

such that:

(C1) For a.e. material point $p \in \Omega, \chi_{p}(t): t \mapsto \chi(p, t)$ is Lipschitz continuous with Lipschitz constant less than or equal to one.

(C2) For a.e. $p \in \Omega, \chi_{p}(0)=S$.

It is tacitly understood that the $\sigma$-algebra of measurable sets of $\Omega$ is complete w.r.t. the probability measure, i.e. that sets contained in a measurable negligible sets are measurable. This is used sometimes in what follows. We shall consider the source point $S \in \mathbb{R}^{N}$ as given and we will denote by $\mathbf{C}_{S}(\Omega)$ the set of all possible $\chi$ 's of $\Omega$ with source $S$.

We remark that in classical continuum mechanics, where $N=3$, (see [12], [23]) the parameter $t$ represents the time variable and, under suitable smoothness assumptions, $\chi$ is a motion of the material body, i.e. a smooth one-parameter family of deformations. Since we are not interested here in studying physical motions, but we inquire into the possible (idealized) shapes of the fluid body with the aim of studying a general variational theory for such shapes, the variable $t$ has only the meaning of a geometric parameter. Therefore there is no risk of confusion in referring to $t$ as time, as we frequently do, after the previous clarification. This time may also be associated with a geodesic distance from the source, in case we think of the set of fibers as a tree or plant and the source as a (thinned) trunk.

Definition 1.1 Given $t \in \mathbb{R}_{+}$, we shall say that two points $p, q \in \Omega$ belong to the same $\chi$-vessel of value $t$ and we will write $p \simeq_{t} q$ if

$$
\chi_{p}(s)=\chi_{q}(s) \quad \text { for all } s \in[0, t] .
$$

For every $t \in \mathbb{R}_{+}$, the equivalence relation $\simeq_{t}$ induces a decomposition of $\Omega$ into equivalence classes $X$. We will call such classes $\chi$-vessels.

Definition 1.2 Given $p \in \Omega$ and $t \in \mathbb{R}_{+}$, the equivalence class of $\simeq_{t}$ which contains $p$ and which will be denoted by $[p]_{t}$ will be named the $\chi$-vessel of the point $p$ at $t$.

Given $\chi \in \mathbf{C}_{S}(\Omega)$ and $t>0$, we shall denote by $\mathcal{V}_{t}(\chi)$ the set of all $\chi$-vessels at $t$, that is,

$$
\mathcal{V}_{t}(\chi):=\Omega / \simeq_{t}
$$

The quotients $V_{t}(\chi)$ for different $t$ arise from different equivalence relations, but there is a natural and obvious inclusion between them. They make a filtration of $\Omega$, since $\forall t^{\prime}>t, \forall p \in \Omega$, $[p]_{t^{\prime}} \subset[p]_{t}$.

We make a few comments in order to explain the above definitions. We are taking a Lagrangian (referential) description of the fluid configurations. At each $t$ the decomposition of $\Omega$ induced by $\simeq_{t}$ corresponds to dividing the body into parts which are mapped, through $\chi$, into tube-like regions of $\mathbb{R}^{N}$ which we are going to identify with rectifiable curves. Since we control only the total amount of fluid carried by these regions, we shrink them to their axial curves. Thus, at each $t$ a set of fibers $\chi$ can be regarded as a set of curves, obtained by varying $[p]_{t}$. Indeed, by Definition 1.1. $\chi_{p}$ coincides on the interval $[0, t]$ with any other function $\chi_{q}$ for $q$ varying in the set $[p]_{t}$. For any $p \in \Omega$ we shall refer to $\chi_{p}$ as the $\chi$-fiber of the point $p$, and for $V=[p]_{t}$ we shall denote by $\chi_{V}$ the function defined on $\left[0, t^{*}\right]$, where $t^{*}=\sup \left\{t \in \mathbb{R}_{+} \mid V \in \mathcal{V}_{t}(\chi)\right\}$, such that $\chi_{V}=\chi_{p}$ on $\left[0, t^{*}\right]$ for every 
$p \in V$; we shall call $\chi_{V}$ the $\chi$-fiber of the $\chi$-vessel $V$. As mentioned above, our discourse can easily be interpreted in another way, as modelling a tree, in which case the $\chi$-vessels represent the branches.

Definition 1.3 Let $\chi \in \mathbf{C}_{S}(\Omega)$. The function $\sigma_{\chi}: \Omega \rightarrow \mathbb{R}_{+}$defined by

$$
\sigma_{\chi}(p):=\inf \left\{t \in \mathbb{R}_{+} \mid \chi_{p}(s) \text { is constant on }[t, \infty[\}\right.
$$

will be called the absorption time. We shall say that a point $p \in \Omega$ is absorbed when $\sigma_{\chi}(p)<\infty$. A point $p \in \Omega$ is absorbed at time $t$ if $\sigma_{\chi}(p) \leqslant t$.

Definition 1.4 Let $\chi \in \mathbf{C}_{S}(\Omega)$ and $t>0$. We shall say that $X \subset \Omega$ is an absorbed set at time $t$ if $\sigma_{\chi}(p) \leqslant t$ for a.e. $p \in X$. If $X \in \mathcal{V}_{t}(\chi)$ is absorbed at $t$, we shall call it an absorbed $\chi$-vessel at $t$. We shall denote by $A_{t}(\chi)$ the set of absorbed points at time $t$.

Definition 1.5 Let $\chi \in \mathbf{C}_{S}(\Omega), t>0$ and $X \in \mathcal{V}_{t}(\chi)$. We shall say that $X$ is a $\chi$-flow at $t$ if it is not an absorbed $\chi$-vessel. We shall denote by $\mathcal{F}_{t}(\chi)$ the set of $\chi$-flows at $t$ and by $F_{t}(\chi)$ the union of all $\chi$-flows at $t$.

REMARK 1.1 We notice that by Definitions 1.4 1.5 every $\chi$-vessel with zero measure is an absorbed $\chi$-vessel, so every $\chi$-flow has a positive outer measure.

DEFINITION 1.6 Let $I \subset \mathbb{R}_{+}$. We shall say that the one-parameter family of sets $V_{t}$ is a $\chi$-vessel evolution if $V_{t} \in \mathcal{V}_{t}(\chi)$ for every $t \in I$ and $V_{t}$ is decreasing under inclusion. In particular, we shall say that $V_{t}$ is a $\chi$-flow evolution if also $V_{t} \in \mathcal{F}_{t}(\chi)$ for every $t \in I$.

Given $V \in \mathcal{V}_{t}(\chi)$, there exists a unique $\chi$-vessel evolution $V_{s}$ for $s \in[0, t]$ such that $V_{t}=V$. The flow evolution is obviously not uniquely determined after $t$, unless we are in the particular case considered in the following definition.

DEFINITION 1.7 We shall say that $V \in \mathcal{F}_{t}(\chi)$ is a non-breaking $\chi$-flow if there is no $s>t$ such that there are $V_{1}, V_{2} \in \mathcal{F}_{S}(\chi)$ with $V_{i} \subset V$ for $i=1,2$ and $V_{1} \neq V_{2}$.

REMARK 1.2 If $V \in \mathcal{F}_{t}(\chi)$ is a non-breaking $\chi$-flow there exists a uniquely determined $\chi$-flow evolution $V_{s}$ for $s \in I$, where $I=\left\{s \geqslant t \mid \exists V^{\prime} \in \mathcal{F}_{s}(\chi), V^{\prime} \subset V\right\}$, such that $V_{t}=V$. We shall refer to $I$ as the survival interval of the non-breaking flow $\chi$.

Definition 1.8 Let $V_{t}$ be a $\chi$-flow evolution for $t$ varying in an interval $I \subset \mathbb{R}_{+}$. We define the dispersion $D_{I}\left(V_{t}\right)$ of $V_{t}$ on $I$ as the set of points $p \in \bigcup_{t \in I} V_{t}$ such that $p \notin \bigcap_{t \in I} V_{t}$ and, for every $t \in I, p$ does not belong to any $\chi$-flow $W \in \mathcal{F}_{t}(\chi), W \neq V_{t}$.

Roughly speaking, the dispersion of $V_{t}$ is the set of points of some $V_{t}$ which leave the flow by stopping or by continuing their motion within a vessel of null measure.

REMARK 1.3 The conditions for $p$ belonging to $D_{I}\left(V_{t}\right)$ can be tested by checking them only on a dense subset of $I$. Moreover, if $V_{t}$ has a non-negligible dispersion on $I$, then $\chi_{V_{b}}$, where $b=\sup (I)$, is not constant on $I$.

Let us introduce the following set:

$$
M_{t}(\chi):=\left\{p \in \Omega \mid \sigma_{\chi}(p)>t\right\}=\Omega \backslash A_{t}(\chi) .
$$

Definition 1.9 Let $\chi \in \mathbf{C}_{S}(\Omega)$ and $t>0$. We shall call the set $S_{t}(\chi)=M_{t}(\chi) \backslash F_{t}(\chi)$ the spread flow at $t$. 
The spread flow at time $t$ is the set of $\chi$-vessels with zero measure which are not made of absorbed fibers. Let us point out some trivial monotonicity properties of the above introduced sets. $F_{t}(\chi)$ and $M_{t}(\chi)$ are decreasing, whereas $A_{\chi}(t)$ is increasing.

Definition 1.10 Let $\chi \in \mathbf{C}_{S}(\Omega)$. We shall say that $\chi$ is a non-spread set of fibers if $\left|S_{t}(\chi)\right|=0$ for every $t \in \mathbb{R}_{+}$.

REMARK 1.4 In order that $\chi$ is a non-spread set of fibers it suffices that $\left|S_{t}(\chi)\right|=0$ for every $t$ in a dense subset $D \subset \mathbb{R}_{+}$. Indeed, suppose that $\left|S_{t}(\chi)\right| \neq 0$ for some $t \in D$. Then $\left|S_{S}(\chi)\right| \neq 0$ for every $s \in[t, t+1 / n]$, otherwise $S_{t}(\chi)$ would have zero measure. Since $D$ is dense in $\mathbb{R}_{+}$, it follows that $D \cap[t, t+1 / n] \neq \emptyset$ and we get a contradiction.

Proposition 1.1 If $\chi \in \mathbf{C}_{S}(\Omega)$ is a measurable mapping with respect to the product measure, then for every $t \in \mathbb{R}_{+}, \chi(\cdot, t)$ is measurable and all the $\chi$-vessels of $\mathcal{V}_{t}(\chi)$ are measurable sets.

Proof. The first claim is true in general for a.e. $t$ (see [13], [10]) and since $\chi(\cdot, t)$ is a continuous function of $t$ it also holds for every $t \in \mathbb{R}_{+}$. The second claim follows from the first one, as we are going to show. Let $p \in \Omega$ and let $D \subset \mathbb{R}_{+}$be a countable dense subset. We remark that $q \in[p]_{t} \in \mathcal{V}_{t}(\chi)$ if and only if for every $s \in D$ with $s \leqslant t, q \in \chi(\cdot, s)^{-1}\left(\chi_{p}(s)\right)$, which is a measurable set. So $[p]_{t}=\bigcap_{s} \chi(\cdot, s)^{-1}\left(\chi_{p}(s)\right)$, and the assertion holds.

Definition 1.11 Let $t \in \mathbb{R}_{+}$and let $D \subset \mathbb{R}_{+}$be a dense subset. For any $\varepsilon>0$, we take a finite set of increasing values $t_{k} \in D$, for $k=1, \ldots, n$, with $t_{1}<\varepsilon, t-\varepsilon<t_{n}<t$ and $\varepsilon<t_{k+1}-t_{k}<2 \varepsilon$. We set $\chi_{t}: p \mapsto \chi(p, t)$ and

$$
\chi_{t}^{\varepsilon}(p)= \begin{cases}\chi_{t_{n}}(p) & \text { if } p \in F_{t_{n}}(\chi), \\ \chi_{t_{k}}(p) & \text { if } p \in F_{t_{k}}(\chi) \backslash F_{t_{k+1}}(\chi), \text { for } k=1, \ldots, n-1, \\ S & \text { if } p \notin F_{t_{1}}(\chi) .\end{cases}
$$

We shall refer to $\chi_{t}^{\varepsilon}$ as an $\varepsilon$-approximation on $D$ of the mapping $\chi_{t}: p \mapsto \chi(p, t)$.

REMARK 1.5 It is easy to see that $\left\|\chi_{t}^{\varepsilon}-\chi_{t}\right\|_{\infty}<2 \varepsilon$ and so $\chi_{t}^{\varepsilon} \rightarrow \chi_{t}$ as $\varepsilon \rightarrow 0$. The key point for proving this is to observe that if $p \notin F_{t_{1}}(\chi)$ then either $p \in S_{t_{1}}(\chi)$ or $p$ is absorbed before $t_{1}$. Because of the non-spread hypothesis, $S_{t}(\chi)$ has zero measure for all $t$ so that for almost every $p \notin F_{t_{1}}(\chi), p$ is absorbed before $t_{1}$. Since $t>t_{n}>t_{1}, \chi(p, t)=\chi\left(p, t_{1}\right)$ because $p$ is absorbed before $t_{1}$. Thus,

$$
\left\|\chi_{t}^{\varepsilon}(p)-\chi_{t}(p)\right\|=\left\|S-\chi_{t}\right\|=\|\chi(p, 0)-\chi(p, t)\|=\left\|\chi(p, 0)-\chi\left(p, t_{1}\right)\right\| \leqslant t_{1}
$$

because $\chi$ is 1-Lipschitz. For the other cases, similar arguments apply. For almost every $p \in F_{t_{k}}(\chi) \backslash$ $F_{t_{k+1}}(\chi), p$ is absorbed before $t_{k+1}$ so that $\chi(p, t)=\chi\left(p, t_{k+1}\right)$. Thus,

$$
\left\|\chi_{t}^{\varepsilon}(p)-\chi_{t}(p)\right\|=\left\|\chi\left(p, t_{k}\right)-\chi(p, t)\right\|=\left\|\chi\left(p, t_{k}\right)-\chi\left(p, t_{k+1}\right)\right\| \leqslant 2 \epsilon .
$$

Proposition 1.2 Let $\chi \in \mathbf{C}_{S}(\Omega)$ be a non-spread set of fibers. If there exists a dense subset $D \subset \mathbb{R}_{+}$such that, for every $t \in D$, every $\chi$-flow $V \in \mathcal{F}_{t}(\chi)$ is a measurable set, then $\chi_{t}=\chi(\cdot, t)$ is a measurable function for every $t \in \mathbb{R}_{+}$.

Proof. We first point out that, for every $t \in D, \mathcal{F}_{t}(\chi)$ is at most a countable set. Indeed, for every $t \in \mathbb{R}_{+}, \mathcal{F}_{t}(\chi)$ is a set of disjoint measurable subsets with positive measure. As a consequence, $F_{t}(\chi)$ is measurable; moreover, given $t \in D$, for every subset $A \subset \mathbb{R}^{N}, \chi(\cdot, t)^{-1}(A) \cap F_{t}(\chi)$ is a 
measurable set, since it is a countable union of measurable sets. Let $\chi_{t}^{\varepsilon}$ be an $\varepsilon$-approximation of $\chi_{t}$ on $D$. For every subset $A \subset \mathbb{R}^{N}$, by the previous assertion, $\left(\chi_{t}^{\varepsilon}\right)^{-1}(A)$ is a measurable set and since $\chi_{t}^{\varepsilon} \rightarrow \chi_{t}$ as $\varepsilon \rightarrow 0, \chi_{t}$ turns out to be a measurable function as a pointwise limit of measurable functions.

REMARK 1.6 We notice that $\left|S_{t}(\chi)\right|=0$ is essential to the measurability of $\chi(\cdot, t)$. Indeed, if $\left|S_{t}(\chi)\right| \neq 0$, even if for every $t \in \mathbb{R}_{+}$all the $\chi$-vessels $V \in \mathcal{V}_{t}(\chi)$ are measurable sets and $S_{t}(\chi)$ is also measurable, we cannot expect $\chi(\cdot, t)$ to be a measurable function, as we can see in the following example. Take $S=0$, the origin of the plane, and consider a segment $I$, of length $1 / 2$, orthogonal to the $x$-axis with middle point $(0,1 / 2)$. Let $V$ be a Vitali non-measurable subset of $I$ (see [13]) and define

$$
\chi(x, t)= \begin{cases}t x & \text { if } x \in V, \\ -t x & \text { if } x \in I \backslash V,\end{cases}
$$

with $\Omega=I$. All the $\chi$-vessels for $t \neq 0$ are negligible (and therefore measurable), so there are no $\chi$-flows, and $S_{t}(\chi)=I$ is measurable. However $\chi$ is clearly not measurable.

LEMma 1.1 Let $f: \Omega \times \mathbb{R}_{+} \rightarrow \mathbb{R}$ be such that $f(\cdot, t)$ is measurable for $t$ in a dense subset $D \subset \mathbb{R}_{+}$and $f(p, \cdot)$ is continuous for a.e. $p \in \Omega$. Then $f$ is a measurable mapping.

Proof. We give the proof of this well-known lemma for the sake of completeness (see e.g. [7]). Let $a, b \in D$. For any $c>0$ and $\varepsilon>0$, we introduce the sets $U=\left\{(p, t) \in \Omega \times \mathbb{R}_{+} \mid f(p, t)>c\right\}$ and $V_{\varepsilon}(a, b)=\{p \in \Omega \mid f(p, s)>c+\varepsilon \forall s \in[a, b] \cap D\}$. We are going to show that, modulo a negligible set,

$$
U=\bigcup_{\substack{a, b \in D \\ \varepsilon \in D}} V_{\varepsilon}(a, b) \times[a, b] .
$$

In order to prove the inclusion $\bigcup V_{\varepsilon}(a, b) \times[a, b] \subset U$, we notice that if $(p, t) \in V_{\varepsilon}(a, b) \times[a, b]$ then, for every $s \in[a, b] \cap D, f(p, s)>c+\varepsilon$, and since we can assume $f(p, \cdot)$ is continuous, by passing to the limit we get $f(p, t) \geqslant c+\varepsilon>c$.

Let us show the reverse inclusion: if $f(p, t)>c$ then there exists $\varepsilon>0$ such that $f(p, t)>c+$ $2 \varepsilon$, so, by continuity, we can take $a, b$ in such a way that for every $s \in[a, b]$ we have $f(p, s)>c+\varepsilon$ and $t \in[a, b]$. Now, since $f(\cdot, t)$ is measurable for every $t \in D$, the set $V_{\varepsilon}(a, b)$ is measurable for any fixed $a, b, \varepsilon$, since it is a countable intersection of measurable sets. Therefore the above equality leads to the conclusion of the lemma.

By combining Proposition 1.1, Proposition 1.2 and Lemma 1.1, we get the following claim.

Proposition 1.3 Let $\chi \in \mathbf{C}_{S}(\Omega)$ be a non-spread set of fibers. Then $\chi$ is measurable if and only if for every $t$ in a dense subset $D \subset \mathbb{R}_{+}$every $\chi$-flow $V \in \mathcal{F}_{t}(\chi)$ is a measurable set and if and only if for every $t \in \mathbb{R}_{+}$every $\chi$-vessel $V \in \mathcal{V}_{t}(\chi)$ is a measurable set.

The previous results can be summarized in the following theorem. Although they cannot be directly deduced from any reference known to us, the results obviously fall in the general framework of time dependent random functions and continuous martingales as studied (e.g.) in [17].

THEOREM 1.1 For every set of fibers $\chi \in \mathbf{C}_{S}(\Omega)$ the following statements are equivalent.

1. $\chi$ is measurable.

2. $\chi(\cdot, t)$ is measurable for every $t$ in a dense subset $D \subset \mathbb{R}_{+}$.

3. $\chi(\cdot, t)$ is measurable for every $t \in \mathbb{R}_{+}$. 
If, in addition, $\chi$ is a non-spread set of fibers, then the following items are also equivalent to the three above.

4. Every $\chi$-vessel is measurable for every $t \in \mathbb{R}_{+}$.

5. Every $\chi$-flow is measurable for every $t$ in a dense subset $D \subset \mathbb{R}_{+}$.

DEFinition 1.12 We shall say that $\chi \in \mathbf{C}_{S}(\Omega)$ is an irrigation pattern $\Omega$ if $\Omega$ it is measurable. The set of all irrigation patterns of $\Omega$ will be denoted by $\mathbf{P}_{S}(\Omega)$.

REMARK 1.7 We introduce measurability because we want to define a cost functional which takes the form of an integral.

REMARK 1.8 An irrigation pattern $\chi$ is generally characterized by each one of the first three items of Theorem 1.1, and if $\chi$ is also non-spread, then it can be characterized by all the items.

Proposition 1.4 For every $\chi \in \mathbf{P}_{S}(\Omega)$, the absorption function $\sigma_{\chi}$ is a measurable mapping.

Proof. Let us first recall some obvious properties of measurable functions (see [13], [10]): the difference of two measurable functions is a measurable function, so the set on which two measurable functions agree is measurable and finally the set on which a sequence of measurable functions agree is measurable. We are going to prove that for every $t>0$ the set $A_{t}(\chi)$ is measurable. Let $D \subset \mathbb{R}_{+}$ be any countable dense subset. Since $s \mapsto \chi(p, s)$ is Lipschitz continuous, the condition $\sigma_{\chi}(p) \leqslant t$ is equivalent to saying that for every $s \in D$ with $s \geqslant t, \chi(p, s)=\chi(p, t)$, that is, $(\chi(\cdot, s))_{s \in D}$ is a sequence of functions which agree at $p$. Since, by item 3 of Theorem 1.1, such functions are measurable, the set where they agree is also measurable.

Proposition 1.5 Let $\chi \in \mathbf{P}_{S}(\Omega)$ be a non-spread irrigation pattern. Then, for a.e. $p \in \Omega$ and for every $t<\sigma_{\chi}(p)$, there exists $V \in \mathcal{F}_{t}(\chi)$ such that $p \in V$.

Proof. Let $(p, t)$ be such that the conclusion does not hold. Let $D \subset \mathbb{R}_{+}$be a dense subset. We fix $s \in D$ such that $t<s<\sigma_{\chi}(p)$. Thus $p$ belongs to the spread flow $S_{s}(\chi)$. Since $\left|\bigcup_{s \in D} S_{s}(\chi)\right|=0$, we see that the set of points $p$ for which the conclusion does not hold is a negligible set.

\section{Cost functional, irrigation patterns convergence}

For any given $\chi \in \mathbf{P}_{S}(\Omega)$ and $\left.\alpha \in\right] 0,1[$, we introduce the following density cost function:

$$
\varphi_{\chi}(p, t):(p, t) \mapsto\left|[p]_{t}\right|^{\alpha-1}
$$

(of course $\varphi_{\chi}(p, t)=\infty$ when $\left|[p]_{t}\right|=0$ ). For every $t \in \mathbb{R}_{+}$, the cost functional is defined as

$$
c_{\chi}(t):=\int_{M_{t}(\chi)} \varphi_{\chi}(p, t) \mathrm{d} p
$$

and the total cost is given by

$$
I(\chi):=\int_{\mathbb{R}_{+}} c_{\chi}(t) \mathrm{d} t .
$$

Proposition 2.1 If $\chi \in \mathbf{P}_{S}(\Omega)$ is such that $I(\chi)<\infty$, then $\chi$ is a non-spread irrigation pattern. 
Proof. The assumption $I(\chi)<\infty$ implies that $c_{\chi}(t)<\infty$ for a.e. $t$, and this in turn implies that $\left|S_{t}(\chi)\right|=0$. Indeed, given $t$ we have $\left|S_{t}(\chi) \cap V\right|=0$ for every absorbed $\chi$-vessel $V \in \mathcal{V}_{t}(\chi)$, so the trace of $S_{t}(\chi)$ on the (countable) union of all the $\chi$-vessels of positive measure is a negligible set (see Remark 1.4 . So $\varphi(p, t)=\infty$ a.e. on $S_{t}(\chi)$ and therefore $c_{\chi}(t)=\infty$ when $\left|S_{t}(\chi)\right| \neq 0$.

DEFINITION 2.1 Let $\left(\chi_{n}\right)_{n \in \mathbb{N}} \subset \mathbf{C}_{S}(\Omega)$ be a sequence of sets of fibers of $\Omega$. We shall say that the sequence converges to a set of fibers $\chi$, i.e. $\chi_{n} \rightarrow \chi$, if for a.e. $p \in \Omega,\left(\chi_{n}\right)_{p}(t) \rightarrow \chi_{p}(t)$ for every $t \in \mathbb{R}_{+}$.

PRoposition 2.2 Let $\left(\chi_{n}\right)_{n \in \mathbb{N}} \subset \mathbf{C}_{S}(\Omega)$ be a sequence of sets of fibers of $\Omega$. Then $\chi_{n} \rightarrow \chi$ if and only if $\chi_{n}$ converges to $\chi$ a.e. in $\Omega \times \mathbb{R}_{+}$.

Proof. If $\chi_{n} \rightarrow \chi$ then, by definition, $\left(\chi_{n}\right)_{p}(t) \rightarrow \chi_{p}(t)$ for every $t \in \mathbb{R}_{+}$and for every $p \in \Omega \backslash N_{\Omega}$ with $\left|N_{\Omega}\right|=0$. Therefore $\chi_{n}(p, t) \rightarrow \chi(p, t)$ for every $(p, t) \in\left(\Omega \times \mathbb{R}_{+}\right) \backslash N^{\prime}$ with $N^{\prime}=N_{\Omega} \times \mathbb{R}_{+}$, and since $\left|N^{\prime}\right|=0$ we conclude that $\chi_{n} \rightarrow \chi$ a.e. in $\Omega \times \mathbb{R}_{+}$. Conversely, assume that $\chi_{n} \rightarrow \chi$ for every $(p, t) \in\left(\Omega \times \mathbb{R}_{+}\right) \backslash N^{\prime}$ with $\left|N^{\prime}\right|=0$. Denote by $E_{p}$ the $p$-section of $\Omega \times \mathbb{R}_{+}$and by $N_{p}$ the set such that $N^{\prime} \cap E_{p}=\{p\} \times N_{p}$. The Fubini theorem says that

$$
0=|N|=\iint_{\Omega \times \mathbb{R}_{+}} 1_{N}(p, t) \mathrm{d} p \mathrm{~d} t=\int_{\Omega}\left(\int_{\mathbb{R}_{+}} 1_{N_{p}}(t) \mathrm{d} t\right) \mathrm{d} p .
$$

Then, for almost every $p, \int_{\mathbb{R}_{+}} 1_{N_{p}}(t) \mathrm{d} t=0$, that is to say, $\left|N_{p}\right|=0$. For such values of $p$, $\left(\chi_{n}\right)_{p} \rightarrow \chi_{p}$ for a.e. $t \in \mathbb{R}_{+}$. Since for a.e. $p \in \Omega,\left(\chi_{n}\right)_{p}$ and $\chi_{p}$ are uniformly Lipschitz continuous with respect to $t$, we finally get the assertion.

The above proposition implies that if $\left(\chi_{n}\right)_{n \in \mathbb{N}} \in \mathbf{P}_{S}(\Omega)$ is a sequence of irrigation patterns and $\chi_{n} \rightarrow \chi$, then $\chi \in \mathbf{P}_{S}(\Omega)$.

PROPOSITION 2.3 If $\chi_{n} \rightarrow \chi$, then for a.e. $p \in \Omega$,

$$
\sigma_{\chi}(p) \leqslant \liminf _{n \rightarrow \infty} \sigma_{\chi_{n}}(p) .
$$

Proof. We fix $p \in \Omega$ and $\bar{t}>\liminf _{n \rightarrow \infty} \sigma_{\chi_{n}}(p)$. For infinitely many values of $n$ we have $\chi_{n}(p, s)=$ const for $s \in[\bar{t}, \infty[$. By passing to the limit on the previous values of $n$ we get $\chi(p, s)=$ const for $s \in\left[\bar{t}, \infty\left[\right.\right.$, which implies, by definition, $\sigma_{\chi}(p) \leqslant \bar{t}$.

\section{Irrigation measure}

Let $\chi \in \mathbf{P}_{S}(\Omega)$. We introduce the irrigation function

$$
i_{\chi}(p)=\chi\left(p, \sigma_{\chi}(p)\right),
$$

defined on the measurable set $A_{\chi}$ of absorbed points. We have $i_{\chi}(p)=\lim _{t \rightarrow \infty} \chi(p, t)$ and so $i_{\chi}: A_{\chi} \rightarrow \mathbb{R}^{N}$ is a measurable function, as the pointwise limit of a sequence of measurable functions.

The function $i_{\chi}$ induces the image (push-forward) measure $\mu_{\chi}$ defined by the formula

$$
\mu_{\chi}(A):=\left|i_{\chi}^{-1}(A)\right|
$$

for any Borel set $A \subset \mathbb{R}^{N}$. We shall refer to $\mu_{\chi}$ as the irrigation measure induced by $\chi$. 
LEMMA 3.1 Let $\left(i_{n}\right)_{n \in \mathbb{N}}$ be any sequence of measurable functions from $\Omega$ into $\mathbb{R}^{N}$ and for every $n \in \mathbb{N} \cup\{\infty\}$ let $\mu_{n}$ be the image measure induced by $i_{n}$. If for every $\varepsilon>0$ there exist $i_{n}^{\varepsilon}$ and $i_{\infty}^{\varepsilon}$ such that $\left|\left\{p \in \Omega \mid i_{n}^{\varepsilon}(p) \neq i_{n}(p)\right\}\right|<\varepsilon$ for every $n \in \mathbb{N} \cup\{\infty\}$ and $i_{n}^{\varepsilon} \rightarrow i_{\infty}^{\varepsilon}$ a.e. in $\Omega$, then $\mu_{n} \rightarrow \mu_{\infty}$, i.e.

$$
\int f \mathrm{~d} \mu_{n} \rightarrow \int f \mathrm{~d} \mu_{\infty}
$$

for every $f \in C_{0}\left(\mathbb{R}^{N}\right)$.

Proof. For every $n \in \mathbb{N} \cup\{\infty\}$, let $\mu_{n}^{\varepsilon}$ be the image measure induced by $i_{n}^{\varepsilon}$. First we notice that $\int f \mathrm{~d} \mu_{n}^{\varepsilon}=\int f \circ i_{n}^{\varepsilon} \mathrm{d} x$ and so, by applying the Lebesgue Theorem we get $\int f \circ i_{n}^{\varepsilon} \mathrm{d} x \rightarrow$ $\int f \circ i_{\infty}^{\varepsilon} \mathrm{d} x=\int f \mathrm{~d} \mu_{\infty}^{\varepsilon}$ for every $f \in C_{0}\left(\mathbb{R}^{N}\right)$. Then, for $n$ large enough, $\left|\int f \mathrm{~d} \mu_{n}^{\varepsilon}-\int f \mathrm{~d} \mu_{\infty}\right|$ $\leqslant \varepsilon$. Moreover, for every $n \in \mathbb{N} \cup\{\infty\}$ we have

$$
\left|\int f \mathrm{~d} \mu_{n}-\int f \mathrm{~d} \mu_{n}^{\varepsilon}\right| \leqslant \varepsilon \text { osc } f .
$$

Then, for $n$ large enough, we can estimate

$$
\left|\int f \mathrm{~d} \mu_{n}-\int f \mathrm{~d} \mu_{\infty}\right| \leqslant 2 \varepsilon \operatorname{osc} f+\varepsilon .
$$

By taking $\varepsilon$ arbitrarily small, we get the assertion.

LEMMA 3.2 Let $\left(\chi_{n}\right)_{n \in \mathbb{N}}$ be a sequence of irrigation patterns such that $\sup _{n} I\left(\chi_{n}\right)<\infty$. If $\chi_{n} \rightarrow$ $\chi_{\infty}$, then for every $\varepsilon>0$ there exist $i_{n}^{\varepsilon}$ and $i_{\infty}^{\varepsilon}$ such that $\left|\left\{p \in \Omega \mid i_{n}^{\varepsilon}(p) \neq i_{\chi_{n}}(p)\right\}\right|<\varepsilon$ for every $n \in \mathbb{N} \cup\{\infty\}$ and $i_{n}^{\varepsilon} \rightarrow i_{\infty}^{\varepsilon}$ a.e. in $\Omega$.

Proof. We begin by showing that there exists a constant $c>0$ such that, for every $n \in \mathbb{N}$ and $t \in \mathbb{R}_{+},\left|M_{t}\left(\chi_{n}\right)\right|<c t^{-1}$. Indeed, the condition $\sup _{n} I\left(\chi_{n}\right)<\infty$ implies that, for every $n$ and $t$, $\int_{0}^{t}\left|M_{s}\left(\chi_{n}\right)\right| \mathrm{d} s$ is bounded; moreover $\left|M_{s}\left(\chi_{n}\right)\right|$ is a decreasing function of $s$ and so the assertion follows by integrating over $[0, t]$.

Given $\varepsilon>0$, we fix $t$ in such a way that $c t^{-1}<\varepsilon$. Then $i_{\chi_{n}}(t)-\chi_{n}(\cdot, t) \neq 0$ at most on $M_{\chi_{n}}(t)$, which has measure less than $\varepsilon$. Since $\chi_{n}(\cdot, t) \rightarrow \chi_{\infty}(\cdot, t)$, we get the conclusion.

By combining Lemmas 3.1 and 3.2 , we can state the following result.

THEOREM 3.1 Let $\left(\chi_{n}\right)_{n \in \mathbb{N}}$ be a sequence of irrigation patterns such that $\sup _{n} I\left(\chi_{n}\right)<\infty$. If $\chi_{n} \rightarrow \chi$, then $\mu_{\chi_{n}} \rightarrow \mu_{\chi}$.

REMARK 3.1 In general the above statement does not hold true without the assumption $\sup _{n} I\left(\chi_{n}\right)<\infty$. Indeed, let $S, Q \in \mathbb{R}^{N}$ be two given points, and consider the sequence of irrigation patterns given by

$$
\chi_{n}(p, t)= \begin{cases}S & \text { for } t \leqslant n, \\ (n+1-t) S+(t-n) Q & \text { for } n \leqslant t \leqslant n+1, \\ Q & \text { for } t \geqslant n+1,\end{cases}
$$

for every $n \in \mathbb{N}$. Let $\chi(p, t)=S$ for every $t \in \mathbb{R}_{+}$. We have $I\left(\chi_{n}\right)=n+1, \chi_{n} \rightarrow \chi$, but $i_{n}$ is the constant function of value $Q$ for every $n$ and $i$ is the constant function of value $S$. 


\section{Irrigation pattern reconstruction}

In this section we shall show that a non-spread irrigation pattern can be reconstructed from the knowledge of the flows and their fibers for a dense subset of the values of the parameter.

Lemma 4.1 Let $\chi, \chi^{\prime} \in \mathbf{P}_{S}(\Omega)$ be two non-spread irrigation patterns such that, for every $t$ in a dense subset $D \subset \mathbb{R}_{+}, \mathcal{F}_{t}(\chi)=\mathcal{F}_{t}\left(\chi^{\prime}\right)$ and $\chi_{V}=\chi_{V}^{\prime}$ on $[0, t]$ for every $V \in \mathcal{F}_{t}(\chi)$. Then $\sigma_{\chi}=\sigma_{\chi^{\prime}}$ a.e.

Proof. We can assume $D$ to be a countable set. For $s \in D$, let

$$
V(s)=\left\{p \in \Omega \mid \sigma_{\chi}(p)<s<\sigma_{\chi^{\prime}}(p) \text { and } \chi_{p}^{\prime} \text { is not constant on }\left[\sigma_{\chi}(p), s\right]\right\} .
$$

Then, modulo a negligible set, $\left\{p \in \Omega \mid \sigma_{\chi}(p)<\sigma_{\chi^{\prime}}(p)\right\}=\bigcup_{s \in D} V(s)$. We claim that $|V(s)|=0$ for every $s \in D$. Indeed, for $s \in D$ fixed, and every $p \in V(s)$, if $\chi^{\prime}$ is non-spread, then $p$ belongs to a negligible set $N_{s}$ or to a $\chi^{\prime}$-flow $V \in \mathcal{F}_{s}\left(\chi^{\prime}\right)$, as stated in Proposition 1.5 . Then $V$ is also a $\chi$-flow and $\chi_{V}=\chi_{V}^{\prime}$ on $[0, s]$, so $\chi_{p}=\chi_{p}^{\prime}$ on $[0, s]$, in contradiction to the definition of $V(s)$. So $V(s) \subset N_{s}$ and therefore $|V(s)|=0$. Thus $\left|\bigcup_{s} V(s)\right|=0$ and so, if $\chi^{\prime}$ is non-spread, then $\sigma_{\chi^{\prime}} \leqslant \sigma_{\chi}$ almost everywhere. If both $\chi$ and $\chi^{\prime}$ are non-spread then, by the last inequality, we get $\sigma_{\chi}=\sigma_{\chi^{\prime}}$.

Proposition 4.1 Let $\chi, \chi^{\prime} \in \mathbf{P}_{S}(\Omega)$ be two non-spread irrigation patterns such that, for every $t$ in a dense subset $D \subset \mathbb{R}_{+}, \mathcal{F}_{t}(\chi)=\mathcal{F}_{t}\left(\chi^{\prime}\right)$ and $\chi_{V}=\chi_{V}^{\prime}$ on $[0, t]$ for every $V \in \mathcal{F}_{t}(\chi)$. Then $\chi=\chi^{\prime}$ a.e.

Proof. For a.e. $p \in \Omega$, by applying Proposition 1.5 and Lemma 4.1, we find that for every $t \in D$ with $t<\sigma_{\chi}(p)=\sigma_{\chi^{\prime}}(p)$ there exists $V \in \mathcal{F}_{t}(\chi)=\mathcal{F}_{t}\left(\chi^{\prime}\right)$ such that $p \in V$ and $\chi(p, t)=$ $\chi^{\prime}(p, t)$. By passing to the limit, the previous equality still holds for $t=\sigma_{\chi}(p)=\sigma_{\chi^{\prime}}(p)$, and by definition, it also holds for $t>\sigma_{\chi}(p)=\sigma_{\chi^{\prime}}(p)$. Thus, $\chi=\chi^{\prime}$ a.e.

For every $t \in \mathbb{R}_{+}$let $\mathcal{F}_{t}$ be a set of subsets of $\Omega$, determined modulo a negligible set, and for every $V \in \mathcal{F}_{t}$ let $f_{V}^{t}$ be a Lipschitz continuous function, with a Lipschitz constant less than or equal to one, from $[0, t]$ to $\mathbb{R}^{N}$ and such that $f_{V}^{t}(0)=S$. We introduce the following compatibility conditions:

(P1) For every $t$ in a dense subset $D \subset \mathbb{R}_{+}, \mathcal{F}_{t}$ is a set of disjoint non-negligible measurable sets $V_{i}$ and $f_{V_{i}}^{t} \neq f_{V_{j}}^{t}$ for $i \neq j$.

(P2) If $t_{1}<t_{2}$, then for every $V \in \mathcal{F}_{t_{2}}$ there exists $W \in \mathcal{F}_{t_{1}}$ such that $V \subset W$ and $f_{V}^{t_{2}}$ is an extension of $f_{W}^{t_{1}}$.

Given any $V \in \mathcal{F}_{t}$, we can by (P1), (P2) define the associated evolution $\left(V_{s}\right)$ for $s \leqslant t$ and the dispersion $D_{I}\left(V_{s}\right)$ on any $I \subset[0, t]$ formally as in Definition 1.8 , by only taking into account the values $t \in D$ (see Remark 1.3).

(P3) Let $V \in \mathcal{F}_{t}$ and let $\left(V_{s}\right)$ be the associated evolution for $s \in I \subset[0, t]$. If $\left|D_{I}\left(V_{s}\right)\right| \neq 0$, then $f_{V_{s}}^{s} \neq$ const on $I$.

(P4) Let $V \in \mathcal{F}_{t}$ and let $\left(V_{s}\right)$ be the associated evolution for $s \in I \subset[0, t]$. Then $V=\bigcap_{s<t} V_{s}$.

(P5) If $V \in \mathcal{F}_{t}$ is a non-breaking set then the mapping $f_{V}^{s}$ is not constant on $[0, s]$ for some $s$ in its survival interval $I$. 
We remark that, given $\chi \in \mathbf{P}_{S}(\Omega)$, if for every $t, \mathcal{F}_{t}=\mathcal{F}_{t}(\chi)$ and $f_{V}^{t}=\chi_{V{ }_{[0, t]}}$ for every $V \in \mathcal{F}_{t}(\chi)$, then the compatibility conditions (P1)-(P5) are satisfied (see Remark 1.3$)$. Conversely, if (P2) holds, we introduce the following notion of reconstructed irrigation pattern by arguing as follows.

For every $t \in D$, let $F_{t}=\bigcup_{V \in \mathcal{F}_{t}} V$. By (P2), the set-valued function $F_{t}$ is decreasing under inclusion with respect to $t$. We can define the function

$$
\sigma(p):=\sup \left\{t \in \mathbb{R}_{+} \mid p \in F_{t}\right\}
$$

and for every $t<\sigma(p)$ we define $\chi_{p}$ to be the fiber of the point $p$ in $[0, t]$ equal to the fiber $f_{V}^{t}$ of $V \in \mathcal{F}_{t}$ such that $p \in V$. Thanks to (P2), $\chi_{p}$ is then coherently defined on $[0, \sigma(p)]$, and by a constant extension on $\left[\sigma(p), \infty\left[\right.\right.$, on all of $\mathbb{R}_{+}$. The required Lipschitz continuity of the fibers is easily verified. By varying $p$ in $\Omega$ we get a set of fibers $\chi$ which we shall call a reconstructed irrigation pattern.

THEOREM 4.1 Assume that the compatibility conditions (P1)-(P5) hold. Then there is a unique non-spread reconstructed irrigation pattern $\chi \in \mathbf{P}_{S}(\Omega)$ such that for every $t \in \mathbb{R}_{+}, \mathcal{F}_{t}=\mathcal{F}_{t}(\chi)$ and $\chi_{V}=f_{V}^{t}$ on $[0, t]$ for every $V \in \mathcal{F}_{t}(\chi)$.

Proof. Let $\chi$ be the reconstructed irrigation pattern and $V \in \mathcal{F}_{t}$. We have to prove that $V \in \mathcal{F}_{t}(\chi)$. Since the points of $V$ have the same fiber on $[0, t], V$ is contained in a $\chi$-vessel at $t$. We claim that $V$ is a $\chi$-vessel. Indeed, let $p \in V$ and $q \notin V$ and let $V_{s}$ be the evolution of $V$ on $[0, t]$. We show that, modulo a negligible set, $q \notin[p]_{t}$. Let $a=\sup \left\{s \in D \mid q \in V_{s}\right\}$. By (P4), since $q \notin V_{t}$, we have $a<t$ and $q \in V_{a}$. Let $I=[a, t]$. If $q \notin D_{I}\left(V_{s}\right)$, then there exists $a<s \leqslant t$ and $W \in \mathcal{F}_{s}$, $W \neq V_{s}$ such that $q \in W$. Then by $(\mathrm{P} 1), \chi_{p}=f_{V_{s}}^{s} \neq f_{W}^{s}=\chi_{q}$ on $[0, s] \subset[0, t]$; so $q \notin[p]_{t}$. Assume $q \in D_{I}\left(V_{s}\right)$. Then $\sigma(q)=a$. If $\left|D_{I}\left(V_{s}\right)\right| \neq 0$, then by (P3), $\chi_{p}=f_{V}^{t}$ is not constant by construction on $[a, t]=[\sigma(q), t]$ and again we have $q \notin[p]_{t}$. Thus the possible exceptions are the points $q$ such that $q \in D_{I}\left(V_{s}\right)$ and $\left|D_{I}\left(V_{s}\right)\right|=0$, where $I$ is defined as above depending on $q$. Now, let $A=\left\{a \in \mathbb{R}_{+}|| D_{a} \mid=0\right\}$ for $I=[a, t]$ and $D_{a}=D_{I}\left(V_{s}\right)$. Then $D=\bigcup_{a \in A} D_{a}$ is negligible and contains $[p]_{t} \backslash V$. It remains to show that $V$ is not an absorbed $\chi$-vessel. We argue as follows. If there exists $s>t$ such that there are $V_{1}, V_{2} \in \mathcal{F}_{s}$ with $V_{1} \neq V_{2}$ and $V_{1}, V_{2} \subset V$, then $f_{V_{1}}^{s} \neq f_{V_{2}}^{s}$ on $[t, s]$ and so at least one of $V_{1}, V_{2}$ is a non-negligible subset of $V$ made of points $q$ such that $\chi_{q}$ is not constant. Otherwise $V$ has a unique evolution $V_{s}$ on its survival interval $I$ and by (P5) we can find a suitable $s \in I, s>t$, such that $f_{V_{s}}^{s}$ is not constant on $[t, s]$ and so $V_{s}$ is a non-negligible subset of $V$ consisting of non-absorbed points. Section 6 shows the lower semicontinuity of the cost functional under pointwise convergence.

Finally, we notice that since any other vessel has an empty intersection with $F_{t}$, it turns out to be an absorbed set, and by Theorem 1.1, we can conclude that $\chi$ is a non-spread irrigation pattern.

\section{Irrigation pattern equivalence}

A natural outcome of the results obtained in the previous section is the choice to consider two irrigation patterns equivalent if their flows have the same measures and the same fibers. More precisely, we make the following definition.

DEFINITION 5.1 Let $\Omega, \Omega^{\prime}$ be two probability spaces. We shall say that two irrigation patterns $\chi \in \mathbf{P}_{S}(\Omega)$ and $\chi^{\prime} \in \mathbf{P}_{S}\left(\Omega^{\prime}\right)$ are equivalent if, for every $t$ in a dense subset $D \subset \mathbb{R}_{+}$, there exists 
a measure preserving bijection $\Psi^{t}: \mathcal{F}_{t}(\chi) \rightarrow \mathcal{F}_{t}\left(\chi^{\prime}\right)$ such that for every $V \in \mathcal{F}_{t}(\chi), \chi_{V}=\chi_{\Psi^{t}(V)}^{\prime}$ on $[0, t]$.

Now we show that the equality of the measures of the flows, required in the previous definition, implies further interesting properties of equivalent irrigation patterns.

Proposition 5.1 Let $\chi, \chi^{\prime} \in \mathbf{P}_{S}(\Omega)$ be two equivalent irrigation patterns. Let $t_{1}<t_{2}, V_{1} \in$ $\mathcal{F}_{t_{1}}(\chi)$ and $V_{2} \in \mathcal{F}_{t_{2}}(\chi)$. If $V_{2} \subset V_{1}$ then $\Psi^{t_{2}}\left(V_{2}\right) \subset \Psi^{t_{1}}\left(V_{1}\right)$.

Proof. Since $V_{2} \subset V_{1}$, it follows that $\chi_{V_{2}}=\chi_{V_{1}}$ on $\left[0, t_{1}\right]$ and so $\chi_{\Psi^{t_{2}}\left(V_{2}\right)}=\chi_{\Psi^{t_{1}}\left(V_{1}\right)}$ on $\left[0, t_{1}\right]$, which in turn implies the assertion.

LEMMA 5.1 Let $\chi, \chi^{\prime}$ be two equivalent irrigation patterns and let $t_{1}<t_{2}$. Then for every $V \in$ $\mathcal{F}_{t_{1}}(\chi)$,

$$
\left|V \backslash F_{t_{2}}(\chi)\right|=\left|\Psi^{t_{1}}(V) \backslash F_{t_{2}}\left(\chi^{\prime}\right)\right| \text {. }
$$

Proof. We notice that for every $W \in \mathcal{F}_{t_{2}}(\chi)$ we have either $W \subset V$ or $W \cap V=\emptyset$, so $V \backslash F_{t_{2}}(\chi)$ can be obtained by subtracting from $V$ its (disjoint) subsets in $\mathcal{F}_{t_{2}}(\chi)$. So, by Definition 5.1 and Proposition 5.1. we can deduce the assertion.

LEMMA 5.2 Let $\chi, \chi^{\prime}$ be two equivalent irrigation patterns. Then for every $t$ in a countable dense subset $D \subset \mathbb{R}_{+}$and for every $V \in \mathcal{F}_{t}(\chi)$ with $\Psi^{t}(V) \in \mathcal{F}_{t}\left(\chi^{\prime}\right)$, we have

$$
\begin{aligned}
\left|A_{t}(\chi) \cap V\right| & =\left|A_{t}\left(\chi^{\prime}\right) \cap \Psi^{t}(V)\right|, \\
\left|M_{t}(\chi) \cap V\right| & =\left|M_{t}\left(\chi^{\prime}\right) \cap \Psi^{t}(V)\right| .
\end{aligned}
$$

Proof. We fix $t>0, V \in \mathcal{F}_{t}(\chi), \varepsilon>0$ and define $V_{\varepsilon}$ as the set of points of $V$ which are absorbed, i.e. lost by the total flow $F_{t}(\chi)$ in the interval $[t, t+\varepsilon]$. That is,

$$
V_{\varepsilon}=V \backslash F_{t+\varepsilon}(\chi) \text {. }
$$

By Lemma 5.1 we know that $\left|V_{\varepsilon}\right|=\left|\left(\Psi^{t}(V)\right)_{\varepsilon}\right|$. Let $t^{\prime}>t$. Then there exists at most one subset $Z\left(t^{\prime}\right) \subset V$ with $Z\left(t^{\prime}\right) \in \mathcal{F}_{t^{\prime}}(\chi)$ such that $\chi Z\left(t^{\prime}\right)$ is constant on $\left[t, t^{\prime}\right]$. It is easy to verify that if there exists such a subset $Z\left(t^{\prime}\right) \subset V$, then $\Psi^{t^{\prime}}\left(Z\left(t^{\prime}\right)\right) \subset \Psi^{t}(V)$ and it satisfies the corresponding condition, that is, $\chi_{\Psi^{t^{\prime}}\left(Z\left(t^{\prime}\right)\right)}^{\prime}$ is constant on $\left[t, t^{\prime}\right]$. Furthermore, we have

$$
\left|Z\left(t^{\prime}\right)\right|=\left|\Psi^{t^{\prime}}\left(Z\left(t^{\prime}\right)\right)\right|
$$

A point $p \in V$ is absorbed if and only if $p \in Z\left(t^{\prime}\right)$ for every $t^{\prime}>t$ or if, for $\varepsilon$ arbitrarily small, there exists $t^{\prime} \in D, t^{\prime}>t$, such that $p \in Z\left(t^{\prime}\right) \subset V$ and $p$ is lost by the total flow in the interval $\left[t^{\prime}, t^{\prime}+\varepsilon\right]$. Thus

$$
A_{t}(\chi) \cap V=\bigcap_{t^{\prime} \in D, t^{\prime}>t} Z\left(t^{\prime}\right) \cup \bigcap_{n \in \mathbb{N}} \bigcup_{t^{\prime}=t+k / 2^{n}, k \in \mathbb{N}}\left(Z\left(t^{\prime}\right)\right)_{1 / n}
$$

By passing to the measures in (5.6) and by using (5.5), we deduce (5.3). To prove (5.4) we notice that $M_{t}(\chi) \cap V=V \backslash\left(A_{t}(\chi) \cap V\right)$ and so, by Definition 5.1 and (5.3), we obtain (5.4).

TheOREM 5.1 Let $\chi, \chi^{\prime} \in \mathbf{P}_{S}(\Omega)$ be two equivalent irrigation patterns. Then $I(\chi)=I\left(\chi^{\prime}\right)$ and $\mu_{\chi}=\mu_{\chi^{\prime}}$. 
Proof. The equality $I(\chi)=I\left(\chi^{\prime}\right)$ easily follows from Definition 5.1 and Lemma 5.2 To prove that $\mu_{\chi}=\mu_{\chi^{\prime}}$, we proceed as follows. Let $n \in \mathbb{N}, \varepsilon=1 / n$, and let $t=n$. We fix a dense set $D \subset \mathbb{R}_{+}$ as in Definition 5.1 and consider an $\varepsilon$-approximation $\chi_{n}^{\varepsilon}$ on $[0, n]$ of $p \mapsto \chi(p, n)$, following Definition 1.11 We denote by $\mu_{n}$ the image measure induced by $\chi_{n}^{\varepsilon}$. Analogously, we consider the corresponding $\varepsilon$-approximation on $[0, n]$ of $p \mapsto \chi^{\prime}(p, n)$ and the corresponding image measure $\mu_{n}^{\prime}$. For all $i$, for $V \in \mathcal{F}_{t_{i}}(\chi)$ and for $B \subset \mathbb{R}^{N}$, let $\mathcal{F}_{t_{i}}^{B}(\chi)=\left\{V \in \mathcal{F}_{t_{i}}(\chi) \mid \chi_{V}\left(t_{i}\right) \in B\right\}$, the flow arriving in $B$ at time $t_{i}$. Then, for every Borel set $B \subset \mathbb{R}^{N}$, we can compute $\mu_{n}(B)$ from the equality

$$
\mu_{n}(B)=\sum_{t_{i} \in D} \sum_{V \in \mathcal{F}_{t_{i}}^{B}(\chi)}\left|V \backslash F_{t_{i+1}}(\chi)\right|,
$$

and $\mu_{n}^{\prime}(B)$ from the analogous formula

$$
\mu_{n}^{\prime}(B)=\sum_{t_{i} \in D} \sum_{V \in \mathcal{F}_{t_{i}}^{B}(\chi)}\left|\Psi^{t_{i}}(V) \backslash F_{t_{i+1}}\left(\chi^{\prime}\right)\right| .
$$

By applying Lemma 5.1 we obtain $\mu_{n}=\mu_{n}^{\prime}$. Finally, by applying Lemma 3.1, we have $\mu_{n} \rightarrow \mu_{\chi}$ and $\mu_{n}^{\prime} \rightarrow \mu_{\chi^{\prime}}$ as $n \rightarrow \infty$. So we finally get $\mu_{\chi}=\mu_{\chi^{\prime}}$.

\section{Semicontinuity properties}

Given $\chi \in \mathbf{P}_{S}(\Omega)$ and $t \in \mathbb{R}_{+}$, we denote by $\chi_{M_{t}(\chi)}$ the characteristic function of $M_{t}(\chi)$, that is,

$$
\chi_{M_{t}(\chi)}(p, t)= \begin{cases}1 & \text { if } \sigma_{\chi}(p)>t \\ 0 & \text { otherwise. }\end{cases}
$$

Lemma 6.1 If $\chi_{n} \rightarrow \chi$, then for a.e. $(p, t) \in \Omega \times \mathbb{R}_{+}$,

$$
\chi_{M_{t}(\chi)}(p, t) \leqslant \liminf _{n \rightarrow \infty} \chi_{M_{t}\left(\chi_{n}\right)}(p, t) .
$$

Proof. We fix $(p, t) \in \Omega \times \mathbb{R}_{+}$and suppose that $\liminf _{n \rightarrow \infty} \chi_{M_{t}\left(\chi_{n}\right)}(p, t)=0$. This means that $\chi_{M_{t}\left(\chi_{n}\right)}(p, t)=0$ for infinitely many values of $n$ and then for such values $\chi_{n}(p, s)$ is constant for all $s \geqslant t$. Since $\chi_{n}(p, s) \rightarrow \chi_{p}(s)$ pointwise in $s$ for a.e. $p$, we see that $\chi_{p}(s)$ is constant for all $s \geqslant t$. Thus $\sigma_{\chi}(p) \leqslant t$ and $\chi_{M_{t}(\chi)}(p, t)=0$.

LEMma 6.2 If $\chi_{n} \rightarrow \chi$, then for a.e. $(p, t) \in \Omega \times \mathbb{R}_{+}$,

$$
\varphi_{\chi}(p, t) \leqslant \liminf _{n \rightarrow \infty} \varphi_{\chi_{n}}(p, t) .
$$

Proof. We fix ( $p, t) \in \Omega \times \mathbb{R}_{+}$and for every integer $n$ we denote by $V_{n}$ the $\chi_{n}$-vessel containing $p$. Let $V=\bigcap_{n} \bigcup_{k \geqslant n} V_{k}$. We claim that $p \simeq_{t} q$ for a.e. $q \in V$. Indeed, if $q \in V$ then $q \in V_{n}$ for infinitely many $n$ and so $\chi_{n}(p, s)=\chi_{n}(q, s)$ for every $s \in[0, t]$. We know that, modulo a negligible set, $\left(\chi_{n}\right)_{q} \rightarrow \chi_{q}$ and this implies $\chi_{q}(s)=\chi_{p}(s)$ for every $s \in[0, t]$, which means $p \simeq_{t} q$. Thus, each element of $V$ is in the $\chi$-vessel containing $p$ so that $|V| \leqslant\left|[p]_{t}\right|$. On the other hand,

$$
|V|=\lim _{n \rightarrow \infty}\left|\bigcup_{k \geqslant n} V_{k}\right| \geqslant \limsup _{n \rightarrow \infty}\left|V_{n}\right| .
$$


Therefore

$$
\begin{aligned}
\varphi_{\chi}(p, t) & \leqslant\left|[p]_{t}\right|^{\alpha-1} \leqslant|V|^{\alpha-1} \\
& \leqslant\left(\limsup _{n \rightarrow \infty}\left|V_{n}\right|\right)^{\alpha-1}=\liminf _{n \rightarrow \infty}\left|V_{n}\right|^{\alpha-1}=\liminf _{n \rightarrow \infty} \varphi_{\chi_{n}}(p, t) .
\end{aligned}
$$

THEOREM 6.1 If $\chi_{n} \rightarrow \chi$, then

$$
I(\chi) \leqslant \liminf _{n \rightarrow \infty} I\left(\chi_{n}\right) .
$$

Proof. By applying the previous two lemmas and the Fatou Lemma, we easily get

$$
\begin{aligned}
I(\chi) & =\int_{\mathbb{R}_{+}} \int_{\Omega}\left(\chi_{M_{t}(\chi)} \varphi_{\chi}\right)(p, t) \leqslant \int_{\mathbb{R}_{+}} \int_{\Omega} \liminf _{n \rightarrow \infty}\left(\chi_{M_{t}\left(\chi_{n}\right)} \varphi_{\chi_{n}}\right)(p, t) \\
& \leqslant \liminf _{n \rightarrow \infty} \int_{\mathbb{R}_{+}} \int_{\Omega}\left(\chi_{M_{t}\left(\chi_{n}\right)} \varphi_{\chi_{n}}\right)(p, t)=\liminf _{n \rightarrow \infty} I\left(\chi_{n}\right) .
\end{aligned}
$$

\section{Obstruction to compactness and histograms}

In general, the class of irrigation patterns defined on $\Omega$ does not enjoy any compactness property. There are trivial examples of sequences of irrigation patterns, even with bounded cost, which have no convergent subsequence. Indeed, for every $n \in \mathbb{N}$ we divide $\Omega=[0,1]$ into $2 n$ increasingly numbered subintervals and we denote by $\Omega_{1}^{n}$ the union of all odd-numbered intervals and by $\Omega_{2}^{n}$ the union of the even-numbered ones. Given $S=0$, we fix $P \in \mathbb{R}^{N}$ such that $|P-0| \leqslant 1$ and for every $t \in \mathbb{R}_{+}$we consider the sequence of irrigation patterns a.e. given by

$$
\chi_{n}(p, t)= \begin{cases}0 & \text { if } p \in \Omega_{1}^{n}, \\ \min (t, 1) P & \text { if } p \in \Omega_{2}^{n},\end{cases}
$$

for $k=0,1, \ldots, 2 n-1$. For every $n \in \mathbb{N}$ we have $I\left(\chi_{n}\right)=2^{-\alpha}$ but compactness fails. Therefore we are forced to check compactness in restricted classes of irrigation patterns. In all that follows, $\Omega=[0,1]$ is endowed with the Lebesgue measure, and with the $\sigma$-algebra of Lebesgue measurable sets. As we shall prove in Lemma 9.1, there is no loss of generality in this assumption. We shall always be able to replace any probability space $[0,1]$ with no atoms by $[0,1]$.

Definition 7.1 We shall say that the irrigation pattern $\chi:[0,1] \times \mathbb{R}_{+} \rightarrow \mathbb{R}^{N}$ is a histogram if for every $t \in \mathbb{R}_{+}$all $V \in \mathcal{F}_{t}(\chi)$ are intervals, modulo a negligible set.

REMARK 7.1 We observe that the condition for an irrigation pattern $\chi:[0,1] \times \mathbb{R}_{+} \rightarrow \mathbb{R}^{N}$ to be a histogram can be tested on a dense subset $D \subset \mathbb{R}_{+}$. This is a simple consequence of the compatibility condition (P4).

The main result in this section relies on the following statement which we shall prove at the end, after some lemmas.

TheOREM 7.1 Let $\chi \in \mathbf{P}_{S}(\Omega)$. Then there exists a histogram pattern $\chi^{\prime}$ equivalent to $\chi$.

Definition 7.2 Let $\chi \in \mathbf{P}_{S}(\Omega)$ and for a fixed $t \in \mathbb{R}_{+}$let $V_{1}, V_{2} \in \mathcal{F}_{t}(\chi)$. We define the separation time $\rho_{\text {sep }}$ between $V_{1}$ and $V_{2}$ as follows:

$$
\rho_{\text {sep }}\left(V_{1}, V_{2}\right):=\sup \left\{s \in \mathbb{R}_{+}, s \leqslant t \mid \exists V \in \mathcal{F}_{s}(\chi): V_{1}, V_{2} \subset V\right\} .
$$


REMARK 7.2 Given $V_{1}, V_{2}, V_{3} \in \mathcal{F}_{t}(\chi)$, we have

$$
\rho_{\text {sep }}\left(V_{1}, V_{2}\right) \geqslant \min \left(\rho_{\text {sep }}\left(V_{1}, V_{3}\right), \rho_{\text {sep }}\left(V_{2}, V_{3}\right)\right) \text {. }
$$

DEFINITION 7.3 Let $\mathcal{G} \subset \mathcal{F}_{t}(\chi)$ be any subset. We shall say that a total order $\preccurlyeq$ in $\mathcal{G}$ is coherent with $\rho_{\text {sep }}$ if the following condition is satisfied: $\rho_{\text {sep }}\left(V_{1}, V_{2}\right) \leqslant \min \left(\rho_{\text {sep }}\left(V_{1}, X\right), \rho_{\text {sep }}\left(V_{2}, X\right)\right)$ for any $V_{1}, X, V_{2} \in \mathcal{G}$ with $V_{1} \preccurlyeq X \preccurlyeq V_{2}$. (Note that by Remark 7.2 the inequality can be replaced by equality.) We shall say that $\mathcal{G}$ endowed with the relation $\preccurlyeq$ coherent with $\rho_{\text {sep }}$ is a coherently ordered set.

LEMMA 7.1 Let $\mathcal{G} \subset \mathcal{F}_{t}(\chi)$ be a coherently ordered set. Then $\preccurlyeq$ can be coherently extended to all of $\mathcal{F}_{t}(\chi)$.

Proof. Let $V \in \mathcal{F}_{t}(\chi) \backslash \mathcal{G}$. We show that $\preccurlyeq$ can be extended to $\mathcal{G} \cup\{V\}$. Let $f: \mathcal{G} \rightarrow \mathbb{R}$. We shall say that $f$ is a concave like function if $f(Y) \geqslant \min (f(X), f(Z))$ for any $X, Y, Z \in \mathcal{G}$ with $X \preccurlyeq Y \preccurlyeq Z$. Now, let $X \in \mathcal{G}$. We define the function $f: X \mapsto \rho_{\text {sep }}(X, V)$ and we notice that since $\preccurlyeq$ is coherent, $f$ is a concave like function. As a consequence, we see that for every $X \in \mathcal{G}$ either of the following two statements holds true:

(a) $\forall Y \in \mathcal{G}$ such that $Y \preccurlyeq X: f(X) \geqslant f(Y)$, or

(b) $\forall Y \in \mathcal{G}$ such that $X \preccurlyeq Y: f(X) \geqslant f(Y)$.

Therefore, if we set

$$
\begin{aligned}
& \mathcal{G}_{1}=\{X \in \mathcal{G} \mid \forall Y \in \mathcal{G}, Y \preccurlyeq X: f(X) \geqslant f(Y)\}, \\
& \mathcal{G}_{2}=\{X \in \mathcal{G} \mid \forall Y \in \mathcal{G}, X \preccurlyeq Y: f(X) \geqslant f(Y)\},
\end{aligned}
$$

then $\mathcal{G}=\mathcal{G}_{1} \cup \mathcal{G}_{2}$. We claim that if $X \in \mathcal{G}_{1}$ and $Y \preccurlyeq X$, then $Y \in \mathcal{G}_{1}$; we shall say that $\mathcal{G}_{1}$ has the left segment property.

Take $Z \preccurlyeq Y$; then $Z \preccurlyeq Y \preccurlyeq X$ so that $f(Z) \leqslant f(X)$ and $f(Y) \geqslant \min (f(X), f(Z))=f(Z)$.

Analogously, if $X \in \mathcal{G}_{2}$ and $X \preccurlyeq Y$ then $Y \in \mathcal{G}_{2}$; we shall say that $\mathcal{G}_{2}$ has the right segment property. Now we extend $\preccurlyeq$ to $\mathcal{G} \cup\{V\}$ by setting $X \preccurlyeq V$ for every $X \in \mathcal{G}_{1}$ and $V \preccurlyeq X$ for every $X \in \mathcal{G} \backslash \mathcal{G}_{1} \subset \mathcal{G}_{2}$. The left segment property of $\mathcal{G}_{1}$ makes such an extension an order. We have to show that such an extension preserves the coherence with $\rho_{\text {sep. }}$. To this end, we fix any three sets $V_{1}, V_{2}, X$ to be tested as in Definition 7.3. Of course, we only have to take care of the case in which one of them is $V$, since $\preccurlyeq$ is already supposed to be coherent on $\mathcal{G}$. The case $V=V_{1}$ is an obvious consequence of the fact that $X \in \mathcal{G}_{2}$ and of the definition of $\mathcal{G}_{2}$. Analogously, the case $V=V_{2}$ is trivial by the definition of $\mathcal{G}_{1}$. When $V=X$, suppose by contradiction that, for $i=1$ or $i=2$, $\rho_{\text {sep }}\left(V_{1}, V_{2}\right)>f\left(V_{i}\right)$, so $f\left(V_{1}\right)=f\left(V_{2}\right)$. We have by construction $V_{1} \in \mathcal{G}_{1}$ and $V_{2} \notin \mathcal{G}_{1}$. Let $W \in \mathcal{G}$ be such that $V_{1} \preccurlyeq W \preccurlyeq V_{2}$. Since $\preccurlyeq$ is coherent in $\mathcal{G}$ we have $\rho_{\text {sep }}\left(W, V_{1}\right) \geqslant \rho_{\text {sep }}\left(V_{1}, V_{2}\right)$ and so $\rho_{\text {sep }}\left(W, V_{1}\right)>f\left(V_{1}\right)$. This inequality obviously implies $f(W)=f\left(V_{1}\right)$ and so $f$ takes the same value on every order interval between $V_{1}$ and $V_{2}$. Therefore, since $V_{1} \in \mathcal{G}_{1}$ and $\preccurlyeq$ is a total order, we get $V_{2} \in \mathcal{G}_{1}$ and this is a contradiction.

Finally, we can conclude that since the property remains true step by step by joining one set at a time and it is of finite type, it holds true for the whole $\mathcal{F}_{t}(\chi)$.

DEFINITION 7.4 Given $t_{1}, t_{2} \in \mathbb{R}_{+}, t_{1}<t_{2}$, let $\mathcal{F}_{t_{i}}(\chi)$ be coherently ordered by $\preccurlyeq t_{i}$ for $i=1,2$. We shall say that $\preccurlyeq_{t_{1}}$ and $\preccurlyeq_{t_{2}}$ are compatible if for any $V_{1}, W_{1} \in \mathcal{F}_{t_{1}}(\chi)$ and $V_{2}, W_{2} \in \mathcal{F}_{t_{2}}(\chi)$ such that $V_{2} \subset V_{1}$ and $W_{2} \subset W_{1}$, if $V_{2} \preccurlyeq t_{2} W_{2}$ then $V_{1} \preccurlyeq_{t_{1}} W_{1}$. In such a case we shall say that $\mathcal{F}_{t_{i}}(\chi)$, for $i=1,2$, are coherently ordered and compatible. 
The following property is a straightforward consequence of the last definition.

LEMMA 7.2 Let $t_{1}<t_{2}<t_{3}$ and let $\mathcal{F}_{t_{i}}(\chi)$ be coherently ordered by $\preccurlyeq t_{i}$ for $i=1,2,3$. If $\mathcal{F}_{t_{1}}(\chi)$ is compatible with $\mathcal{F}_{t_{2}}(\chi)$ and $\mathcal{F}_{t_{2}}(\chi)$ is compatible with $\mathcal{F}_{t_{3}}(\chi)$, then $\mathcal{F}_{t_{1}}(\chi)$ is compatible with $\mathcal{F}_{t_{3}}(\chi)$.

LEMMA 7.3 Let $\mathcal{T} \subset \mathbb{R}_{+}$be a finite set of values and let $\mathcal{F}_{s}(\chi)$ be coherently ordered by $\preccurlyeq s$ and compatible for $s \in \mathcal{T}$. Given any $t \in \mathbb{R}_{+}$there exists a coherent order $\preccurlyeq_{t}$ which is compatible with every $\preccurlyeq s$ for $s \in \mathcal{T}$.

Proof. Let $s=\max \{\rho \in \mathcal{T} \mid \rho<t\}$ and $\tau=\min \{\rho \in \mathcal{T} \mid \rho>t\}$. Given $W \in \mathcal{F}_{s}(\chi)$, we set $\mathcal{F}_{t}(W)=\left\{V \in \mathcal{F}_{t}(\chi) \mid V \subset W\right\}$. By varying $W$, we get different sets of sets each one collecting the $\chi$-flows at $t$ which are contained in the same $\chi$-flow $W$ at the time $s$. Let $\mathcal{G}(W)=$ $\left\{V \in \mathcal{F}_{t}(W) \mid \exists X \in \mathcal{F}_{\tau}(\chi): X \subset V\right\}$. If $V_{1}, V_{2} \in \mathcal{G}(W)$ and $X_{1}, X_{2} \in \mathcal{F}_{\tau}(\chi)$ satisfy $X_{1} \subset V_{1}$, $X_{2} \subset V_{2}$, we define $V_{1} \preccurlyeq_{t} V_{2}$ if $X_{1} \preccurlyeq_{\tau} X_{2}$, that is, we define in $\mathcal{G}(W)$ the coherent order inherited from $\mathcal{F}_{\tau}(\chi)$.

We show that $\preccurlyeq t$ does not depend on the choice of $X_{1}, X_{2}$. Indeed, let $X_{1} \preccurlyeq \tau X_{2}$ and $Y_{2} \preccurlyeq \tau Y_{1}$, with $X_{i}, Y_{i} \subset V_{i}$ for $i=1$, 2. Since $\preccurlyeq_{\tau}$ is a total ordering, we have $X_{2} \preccurlyeq_{\tau} Y_{1}$ or $Y_{1} \preccurlyeq_{\tau} X_{2}$. If $X_{2} \preccurlyeq_{\tau} Y_{1}$ then $X_{1} \preccurlyeq_{\tau} X_{2} \preccurlyeq_{\tau} Y_{1}, \rho_{\text {sep }}\left(X_{1}, Y_{1}\right) \geqslant t$ and $\rho_{\text {sep }}\left(X_{1}, X_{2}\right)<t$ contradicts the coherence of $\preccurlyeq_{\tau}$. Otherwise, a similar argument leads to a contradiction from $Y_{2} \preccurlyeq_{\tau} Y_{1} \preccurlyeq_{\tau} X_{2}$. Notice that $\preccurlyeq_{t}$ is coherent with $\rho_{\text {sep }}$ since for every $V_{1}, V_{2} \in \mathcal{G}(W)$ we have $\rho_{\text {sep }}\left(V_{1}, V_{2}\right)=\rho_{\text {sep }}\left(X_{1}, X_{2}\right)$, where $X_{1}$ and $X_{2}$ are contained respectively in $V_{1}$ and $V_{2}$ at time $\tau$. Thus every $\mathcal{G}(W)$ is endowed with the order $\preccurlyeq_{t}$ inherited from $\preccurlyeq_{\tau}$ and by Lemma 7.1 we can extend $\preccurlyeq_{t}$ to a total ordering on all of $\mathcal{F}_{t}(W)$.

If $V_{1} \in \mathcal{F}_{t}\left(W_{1}\right)$ and $V_{2} \in \mathcal{F}_{t}\left(W_{2}\right)$ and $W_{1} \preccurlyeq s W_{2}$, we set $V_{1} \preccurlyeq_{t} V_{2}$. The reader can easily check that $\preccurlyeq_{t}$ is coherent and compatible with $\preccurlyeq_{\tau}$ and $\preccurlyeq_{s}$. Then, by Lemma 7.2, $\preccurlyeq_{t}$ is compatible with $\preccurlyeq \rho$ for every $\rho \in \mathcal{T}$.

LEMmA 7.4 There exists a dense subset $D \subset \mathbb{R}_{+}$and for every $t \in D$ a coherent ordering $\preccurlyeq_{t}$ such that all the sets of $\mathcal{F}_{t}(\chi)$ are coherently ordered and compatible.

Proof. Let $D \subset \mathbb{R}_{+}$be any countable dense set. By applying Lemma 7.3 we can proceed step by step, defining $\preccurlyeq_{t}$ by adding one value at a time.

Given two intervals $I_{1}, I_{2} \subset \mathbb{R}_{+}$, we shall write $I_{1} \leqslant I_{2}$ if $x \leqslant y$ for every $x \in I_{1}$ and every $y \in I_{2}$. Let $D \subset \mathbb{R}_{+}$be a subset and let $\mathcal{F}_{t}(\chi)$ be coherently ordered for every $t \in D$. Let $\mathcal{I}^{t}=\left(I_{V}\right)_{V \in \mathcal{F}_{t}(\chi)}$ be a family of open disjoint intervals of $[0,1]$ such that $\left|I_{V}\right|=|V|$ and if $V \prec_{t} W$ then $I_{V} \leqslant I_{W}$. We shall refer to $\mathcal{I}^{t}$ as an ordered family of intervals. Let $\mathcal{I}^{1}=\left(I_{V}^{1}\right)_{V \in \mathcal{F}_{t}(\chi)}$ and $\mathcal{I}^{2}=\left(I_{V}^{2}\right)_{V \in \mathcal{F}_{t}(\chi)}$ be two ordered families of intervals. We shall write $\mathcal{I}^{1} \preccurlyeq \mathcal{I}^{2}$ whenever for every $V \in \mathcal{F}_{t}(\chi)$ the middle point of $I_{V}^{1}$ is less than or equal to the middle point of $I_{V}^{2}$.

DEFINITION 7.5 Let $t_{1}, t_{2} \in \mathbb{R}_{+}, t_{1}<t_{2}$, and let $\mathcal{I}^{t_{1}}=\left(I_{V}^{t_{1}}\right)_{V \in \mathcal{F}_{t_{1}}(\chi)}$ and $\mathcal{I}^{t_{2}}=\left(I_{V}^{t_{2}}\right)_{V \in \mathcal{F}_{t_{2}}(\chi)}$ be two ordered families of intervals. We shall say that $\mathcal{I}^{t_{1}}$ and $\mathcal{I}^{t_{2}}$ are compatible if $I_{V_{2}}^{t_{2}} \subset I_{V_{1}}^{t_{1}}$ for any $V_{1} \in \mathcal{F}_{t_{1}}(\chi)$ and $V_{2} \in \mathcal{F}_{t_{2}}(\chi)$ such that $V_{2} \subset V_{1}$.

Let $D \subset \mathbb{R}_{+}$be a given subset and let $\mathcal{F}_{t}(\chi)$ be coherently ordered by $\preccurlyeq_{t}$ and compatible for $t \in D$. If $\mathcal{I}^{t}$ are compatible ordered families of intervals for all $t \in D$, then we shall refer to $\mathbf{I}_{D}:=\left(\mathcal{I}^{t}\right)_{t \in D}$ as a compatible grid. Let $\mathbf{I}_{D}=\left(\mathcal{I}^{t}\right)_{t \in D}$ and $\mathbf{J}_{D}=\left(\mathcal{J}^{t}\right)_{t \in D}$ be two compatible grids. We shall write $\mathbf{I}_{D} \preccurlyeq \mathbf{J}_{D}$ if $\mathcal{I}^{t} \preccurlyeq \mathcal{J}^{t}$ for every $t \in D$. 
Lemma 7.5 Let $\mathcal{T} \subset \mathbb{R}_{+}$be any finite subset and let $\mathcal{F}_{t}(\chi)$ be coherently ordered by $\preccurlyeq_{t}$ and compatible for $t \in \mathcal{T}$. Then there exists a compatible grid $\mathbf{I}_{\mathcal{T}}$ such that $\mathbf{I}_{\mathcal{T}} \preccurlyeq \mathbf{J}_{\mathcal{T}}$ for any other compatible grid $\mathbf{J}_{\mathcal{T}}$.

Proof. Let $t_{i}, i=1, \ldots, n$, be the elements of $\mathcal{T}$ listed in increasing order. We start with $\mathcal{I}^{t_{1}}$ which, being an ordered family of intervals, consists of subintervals of $[0,1]$ having a total measure less than one; we place consecutively such intervals starting from zero. Thus the intervals of $\mathcal{I}^{t_{1}}$ turn out to be arranged in $[0,1]$ to the left as much as possible. Now we pass to $\mathcal{I}^{t_{2}}$ and we put the intervals $I_{j} \in \mathcal{I}^{t_{2}}$ which are contained in the same interval $I \in \mathcal{I}^{t_{1}}$ consecutively and on the left of $I$. We can easily iterate this procedure step by step for all values of $\mathcal{T}$.

Lemma 7.6 Let $D \subset \mathbb{R}_{+}$be a countable subset. Then there exists a compatible grid $\mathbf{I}_{D}$.

Proof. Let $D=\bigcup_{n} \mathcal{T}_{n}$, with $\mathcal{T}_{n} \subset \mathcal{T}_{n+1}$ for every $n \in \mathbb{N}$. For every $n$, we take a compatible grid $\mathbf{I}_{\mathcal{T}_{n}}$ as in Lemma 7.5. We observe that the restriction of $\mathbf{I}_{\mathcal{T}_{n+1}}$ to $\mathcal{T}_{n}$ is a compatible grid. So, by the minimality property of $\mathbf{I}_{\mathcal{T}_{n}}$, we can compare the corresponding intervals of $\mathbf{I}_{\mathcal{T}_{n+1}}$ and $\mathbf{I}_{\mathcal{T}_{n}}$ and the first ones turn out to be at most shifted to the right side of $[0,1]$. Thus, for fixed $t \in D$, we have $t \in \mathcal{T}_{n}$ for every $n$ greater than some $\bar{n}$ and the ordered family of intervals $\mathcal{I}^{t}$ has the intervals at most shifted to the right. We can pass to the limit as $n \rightarrow \infty$ and thanks to the boundedness of $[0,1]$ we get a limit grid $\mathbf{I}_{D}=\left(\mathcal{I}^{t}\right)_{t \in D}$. Now, for every $t \in D$, $\mathcal{I}^{t}$ is an ordered family of intervals and all $\mathcal{I}^{t}$ are compatible and so $\mathbf{I}_{D}$ is a compatible grid.

Proof of Theorem 7.1. Let $\mathbf{I}_{D}=\left(\mathcal{I}^{t}\right)_{t \in D}$ as given by Lemma7.6 and for every $t \in D$ set $\mathcal{F}_{t}=\mathcal{I}^{t}$. Fix $t \in D$. To every $I_{V}^{t} \in \mathcal{I}^{t}$ we associate $f_{V}^{t}=\chi_{\left.V\right|_{[0, t]}}$ and we easily verify that the compatibility conditions (P1)-(P5) are satisfied. Thus for every $t \in D$ the intervals $I_{V}^{t}$ are the flows of an irrigation pattern $\chi^{\prime}$ which is a histogram equivalent to $\chi$.

\section{Compactness properties}

The main result of this section relies on the following compactness theorem which we shall prove after some preliminary lemmas.

THEOREM 8.1 Let $\left(\chi_{n}\right)_{n \in \mathbb{N}}$ be a sequence of histograms with $I\left(\chi_{n}\right)$ bounded. Then there exists a subsequence $\left(\chi_{k_{n}}\right)_{k \in \mathbb{N}}$ converging to an irrigation pattern $\chi$.

In the following we shall often need to pass to a subsequence of a given one, so to keep the notation as simple as possible, we will not use further subscripts, but we will relabel the sequence every time. Therefore, $\left(\chi_{n}\right)_{n \in \mathbb{N}}$ will denote at each step a relabelled suitable subsequence for which all previously proved lemmas simultaneously hold.

The following statement is an easy variant of the Arzelà-Ascoli Theorem.

LEMmA 8.1 Let $\left(\chi_{n}\right)_{n \in \mathbb{N}}$ be a sequence of histograms with $I\left(\chi_{n}\right)$ bounded. Then the sequence admits a converging subsequence $\left(\chi_{k_{n}}\right)_{n \in \mathbb{N}}$ such that $\left(\chi_{k_{n}}\right)_{p} \rightarrow \chi_{p}$ for every $p \in \mathbb{Q} \cap[0,1]$.

LEMMA 8.2 Let $\left(\chi_{n}\right)_{n \in \mathbb{N}}$ be a sequence of histograms with $I\left(\chi_{n}\right)$ bounded. Then, upon passing to a subsequence, we can find a countable dense subset $D \subset \mathbb{R}_{+}$such that $\left(c_{\chi_{n}}(t)\right)_{n \in \mathbb{N}}$ is bounded on $D$.

Proof. Indeed,

$$
\int_{\mathbb{R}_{+}} \liminf _{n \rightarrow \infty} c_{\chi_{n}}(t) \leqslant \liminf _{n \rightarrow \infty} \int_{\mathbb{R}_{+}} c_{\chi_{n}}(t)=\liminf _{n \rightarrow \infty} I\left(\chi_{n}\right)<\infty
$$


and so $\liminf _{n \rightarrow \infty} c_{\chi_{n}}(t)<\infty$ for a.e. $t$, which in turn implies that for a.e. $t \in \mathbb{R}_{+}, c_{\chi_{n}}(t)$ has a bounded subsequence. Then, by a diagonal selection argument we get a bounded sequence $\left(c_{\chi_{n}}(t)\right)_{n \in \mathbb{N}}$ for $t$ in a countable dense subset $D \subset \mathbb{R}_{+}$.

We fix $D$ as in Lemma 8.2. Let $t \in D$ and $k \in \mathbb{N}$ be fixed and for every index $n$ let

$$
\mathcal{F}_{t}^{k}\left(\chi_{n}\right)=\left\{V \in \mathcal{F}_{t}\left(\chi_{n}\right)|| V \mid \geqslant 1 / k\right\} .
$$

We take a subsequence of $\left(\chi_{n}\right)_{n \in \mathbb{N}}$ in such a way that every sequence $\left(V_{n}\right)_{n \in \mathbb{N}}$ of flows selected from $\mathcal{F}_{t}^{k}\left(\chi_{n}\right)$ converges to some interval $V$ contained in $[0,1]$ as $n \rightarrow \infty$ (there are finitely many such intervals). By Lemma 8.1 we have $\chi_{V_{n}} \rightarrow f_{V}^{t}$ on $[0, t]$ with $f_{V}^{t}=\chi_{p}$ for every $p \in \mathbb{Q} \cap \stackrel{\circ}{V}$. By a diagonal selection we pass to a subsequence for which the previous convergence is true for every integer $k$ and for every $t \in D$. We denote by $L(t)$ the union of the open limit intervals determined for a given $t \in D$.

LEMMA 8.3 With the above notation we also have, for every $t \in D$,

$$
\lim _{n \rightarrow \infty}\left|M_{t}\left(\chi_{n}\right) \backslash L(t)\right|=0
$$

Proof. Fix $k \in \mathbb{N}$ and, for every integer $n$, let $L_{n}^{k}(t)=\bigcup_{V \in \mathcal{F}_{t}^{k}\left(\chi_{n}\right)} V$. Since each of these flows converges to a limit interval contained in $L(t)$, we have $\left|L_{n}^{k}(t) \backslash L(t)\right| \rightarrow 0$ as $n \rightarrow \infty$. Moreover, $\varphi_{\chi_{n}}(p, \cdot) \geqslant k^{1-\alpha}$ for a.e. $p \in M_{t}\left(\chi_{n}\right) \backslash L_{n}^{k}(t)$. Therefore, $\left|M_{t}\left(\chi_{n}\right) \backslash L_{n}^{k}(t)\right| \leqslant k^{\alpha-1} c_{\chi_{n}}(t)$, and since $c_{\chi_{n}}(t)$ is bounded for $t \in D$, by taking $k$ arbitrarily large, we reach the conclusion.

LEMMA 8.4 Upon replacing $\left(\chi_{n}\right)_{n \in \mathbb{N}}$ by a suitable subsequence, for every $t \in D$ there exists a negligible set $N(t)$ such that

$$
\bigcap_{n} \bigcup_{k \geqslant n} M_{t}\left(\chi_{k}\right) \subset L(t) \cup N(t) .
$$

Proof. Indeed, $\mathbf{1}_{M_{t}\left(\chi_{n}\right) \backslash L(t)} \rightarrow 0$ in $L^{1}([0,1])$ by Lemma 8.3 so we can take a subsequence converging to zero off a negligible set $N(t)$. Then for every $p \notin L(t) \cup N(t)$ we have $\mathbf{1}_{M_{t}\left(\chi_{n}\right)}(p)=0$ for $n$ large enough. Through a diagonal selection we can get a suitable $\chi_{n}$ which simultaneously satisfies the property for every $t \in D$.

Proof of Theorem 8.1. For every $t \in D$ let $\mathcal{F}_{t}$ be the set of limit open intervals to which all the non-vanishing $\chi_{n}$-flows converge, let $L(t)=\bigcup_{V \in \mathcal{F}_{t}} V, \sigma(p)=\sup \left\{t \in \mathbb{R}_{+} \mid p \in L(t)\right\}$ and for $V \in \mathcal{F}_{t}$ let $f_{V}^{t}$ be the relevant limit fiber. Since the set $\mathcal{F}_{t}$ and the fibers $f_{V}^{t}$ satisfy the compatibility condition (P2), as is easy to verify, we can define the reconstructed irrigation pattern $\chi$. We have to prove that $\chi_{n} \rightarrow \chi$ for a.e. $p \in[0,1]$. To this end, let $N=\bigcup_{t \in D} N(t)$, obtained in Lemma 8.4. and fix $p \in[0,1] \backslash N$. Let $t \in D$ with $t<\sigma(p)$. Then $p$ is an inner point for one of the limit intervals $V \in \mathcal{F}_{t}$ and so there exists $\varepsilon>0$ such that $[p-\varepsilon, p+\varepsilon] \subset V$ and, for a suitably large $n \in \mathbb{N},[p-\varepsilon, p+\varepsilon] \subset V_{n}$ with $V_{n} \in \mathcal{F}_{t}\left(\chi_{n}\right)$ and $V_{n} \rightarrow V$. Let $q \in \mathbb{Q} \cap[p-\varepsilon, p+\varepsilon]$. Then for every integer $n,\left(\chi_{n}\right)_{p}=\left(\chi_{n}\right)_{q}$, we know that $\left(\chi_{n}\right)_{q}=\left(\chi_{n}\right)_{V_{n}}$ and $\left(\chi_{n}\right)_{V_{n}} \rightarrow f_{V}^{t}=\chi_{p}$ on $[0, t]$, thus $\left(\chi_{n}\right)_{p} \rightarrow \chi_{p}$ on $[0, \sigma(p)]$. If $t>\sigma(p)$, then since $p \notin N(t) \cup L(t)$, by Lemma 8.4 for $n$ large enough, $p \notin M_{t}\left(\chi_{n}\right)$. Therefore $\left(\chi_{n}\right)_{p}$ is constant on $[t, \infty[$. Thus the uniform distance between $\left(\chi_{n}\right)_{p}$ and the constant function $\chi_{n}(p, \sigma(p))$ is less than or equal to $|t-\sigma(p)|$, which can be taken arbitrarily small on $\left[\sigma(p), \infty\right.$ [ by the Lipschitz continuity of $\chi_{n}$. Then $\left(\chi_{n}\right)_{p}$ uniformly converges to $\chi(p, \sigma(p))=\chi_{p}$ on $[\sigma(p), \infty[$. Finally, $\chi$ is an irrigation pattern since it is a set of fibers and it is the pointwise limit of a sequence of irrigation patterns. 


\section{Minimization problems on irrigation patterns}

The compactness properties in the previous section, the possibility of taking minimizing sequences among the histograms guaranteed by the results in Section 8 , the semicontinuity properties in Section 6 and the weak continuity of the irrigation measure $\mu_{\chi}$ established in Section 3 , enable us to easily find a solution of the minimization problem

$$
\min _{\chi \in \mathbf{P}_{S}(\Omega)} E(\chi),
$$

for $\Omega=[0,1]$, in which $E(\chi)$ is a functional of the type

$$
E(\chi)=I(\chi)+J\left(\mu_{\chi}\right)
$$

where $J: \mathcal{M}_{+}\left(\mathbb{R}^{N}\right) \rightarrow \mathbb{R}$ is a weakly lower semicontinuous functional defined on the space $\mathcal{M}_{+}\left(\mathbb{R}^{N}\right)$ of positive finite Radon measures in $\mathbb{R}^{N}$, provided we are able to find any irrigation pattern $\chi \in \mathbf{P}_{S}(\Omega)$ such that $I(\chi)<\infty$ and $J\left(\mu_{\chi}\right)<\infty$. This last condition is not a completely trivial requirement and we refer the reader to [29], [5], [6] for more information on this point.

By the results of Section 8, we can say that the minimum irrigation pattern can be taken as a histogram. More generally, the minimization problem 9.9 can be solved for a general $\mu$ measurable set $\Omega$ where $\mu$ is a measure with no atom. This immediately follows by the next simple lemma which shows how a non-atomic measure can be transported to yield the Lebesgue measure.

LEMMA 9.1 Let $\Omega$ be a measurable set equipped with a probability measure $\mu$ with no atom. Then there exists a measurable mapping $T: \Omega \rightarrow[0,1]$ such that $T \# \mu(B)=|B|$ for every Borel set $B \subset[0,1]$, where $T \# \mu$ is the image measure induced by $T$.

Proof. By the Lyapunov theorem, the no-atom condition permits us to split any measurable subset of the $\Omega$ into two parts with equal measure. We define recursively a sequence $\left(T_{n}\right)_{n \in \mathbb{N}}$ of mappings $T_{n}: \Omega \rightarrow[0,1]$. We set $T_{0}=0$ and, given $T_{n}$, we divide its level sets in two parts of equal measure; then we define $T_{n+1}$ by adding to $T_{n}$ the quantity $2^{-n}$ only on one of such parts. Then $T_{n} \# \mu$ turns out to be the sum of $2^{n}$ equally spaced Dirac masses in $[0,1]$. By monotonicity $T_{n}$ uniformly converges to a function $T$. By Lemma 3.1. $T \# \mu$ is the weak limit of the sequence $\left(T_{n} \# \mu\right)_{n \in \mathbb{N}}$ and so it is the Lebesgue measure.

For any fixed $\Omega$ as above, we set $m(\Omega)=\inf _{\chi \in \mathbf{P}_{S}(\Omega)} E(\chi)$. The previous results allow us to state that, given $\chi \in \mathbf{P}_{S}([0,1])$ and any set $\Omega, \chi(T(p), t)$ defines an equivalent irrigation pattern belonging to $\mathbf{P}_{S}(\Omega)$, and this in turn allows us to deduce that

$$
m(\Omega) \leqslant m([0,1]) .
$$

Now, thanks to the compactness results mentioned at the beginning of this section, we can find a histogram $\chi^{\prime}$ such that $E\left(\chi^{\prime}\right) \leqslant m(\Omega)$ and thus we obtain

$$
m(\Omega)=m([0,1]),
$$

so $m(\Omega)$ does not depend on the particular choice of $\Omega$. Finally, by Lemma 9.1 we can exhibit a minimum irrigation pattern on every set $\Omega$.

A case of particular interest is given by

$$
J\left(\mu_{\chi}\right)= \begin{cases}0 & \text { if } \mu_{\chi}=\bar{\mu} \\ \infty & \text { otherwise }\end{cases}
$$


for a given $\bar{\mu} \in \mathcal{M}_{1}\left(\mathbb{R}^{N}\right)$, which leads to the minimization problem with boundary condition $\mu_{\chi}=\bar{\mu}$.

More generally, we can consider systems of finitely (or even countably) many irrigation patterns $\chi_{1}, \ldots, \chi_{n}$ by minimizing functionals of the type

$$
E\left(\chi_{1}, \ldots, \chi_{n}\right)=\sum_{i=1}^{n} I\left(\chi_{i}\right)+J_{1}\left(\mu_{\chi_{1}}, \ldots, \mu_{\chi_{n}}\right)+J_{2}\left(S_{1}, \ldots, S_{n}\right),
$$

where $S_{1}, \ldots, S_{n} \in \mathbb{R}^{N}$ are the source points respectively of $\chi_{1}, \ldots, \chi_{n}$ and $J_{2}$ is a lower semicontinuous function. Among those problems we point out two cases of special interest in which $n=2$ and which can be included in a more general class of transmission problems:

(TP1) Porous systems like those involving lungs and bronchial trees could be modelled in this setting. Here

$$
J_{1}\left(\mu_{\chi_{1}}, \mu_{\chi_{2}}\right)=J_{0}\left(\mu_{\chi_{1}}\right)+\tilde{J}\left(\mu_{\chi_{1}}, \mu_{\chi_{2}}\right),
$$

where

$$
\tilde{J}\left(\mu_{\chi_{1}}, \mu_{\chi_{2}}\right)= \begin{cases}0 & \text { if } \mu_{\chi_{1}}=\mu_{\chi_{2}} \\ \infty & \text { otherwise. }\end{cases}
$$

(TP2) Systems involving two networks with a common source like source-mouth in the case of rivers, or root-foliage in the case of trees can be cast in this formulation. Here

$$
J_{2}\left(S_{1}, S_{2}\right)=J_{0}\left(S_{1}\right)+\bar{J}\left(S_{1}, S_{2}\right),
$$

where

$$
\bar{J}\left(S_{1}, S_{2}\right)= \begin{cases}0 & \text { if } S_{1}=S_{2} \\ \infty & \text { otherwise }\end{cases}
$$

Now a clarification concerning the structural condition (C2) fixing the source $S$ of a set of fibers is in order. The elimination of $(\mathrm{C} 2)$ would introduce the need of further conditions to test the measurability of $\chi$ and would not lead to any substantial advantage, since the cost of an irrigation pattern with many sources is an additive function. In this perspective, the last systems can be considered with any arbitrary number of irrigation patterns by adding a boundary condition involving all the sources. We can pictorially explain this point by saying that, thanks to the additivity property, the tree model can be employed for a forest. Such a model can be interpreted as a transport path (see [29]). We refer the reader to the works [16], [15], [21], [11], [9] and to the surveys [1], [8], [24] for an account of the broad subject of optimal transport problems.

\subsection{The Eulerian and Lagrangian approaches}

It is now time to compare the results presented in this paper with the Eulerian treatment given by Qinglan Xia. He starts with finite atomic measures $a$ and $b$ and defines a "path" from $a$ to $b$ as a flow on a finite embedded graph whose vertices either have at least one arriving edge and one departing edge, or belong to $a$ or $b$. He denotes by $e$ the (straight) edges of the graph, by $w(e)$ the flow inside, and by $\vec{e}$ a unit vector oriented by $e$. He writes $[e]=\mathcal{H}_{\mid}^{1} \vec{e}$ for the vector measure obtained as the product of the Hausdorff measure restricted to $e$ and of the vector $\vec{e}$. Then the embedded path from $a$ to $b$ can be written as the vector measure

$$
G=\sum_{e} w(e)[[e]] .
$$


With this simple formalism, the Kirchhoff law can be expressed in a synthetic way as

$$
\operatorname{div}(G)=a-b .
$$

Letting $\mathcal{G}_{\Lambda}$ be the set of all such paths between atomic measures $a$ and $b$ in a compact and convex subset of $\mathbb{R}^{n}$, Xia defines on $\mathcal{G}_{\Lambda}$ the cost functional

$$
M^{\alpha}(G)=\sum_{e} w(e)^{\alpha} \text { length }(e) .
$$

Then he proceeds to define transport paths connecting two Radon measures $\mu^{+}$and $\mu^{-}$with equal total mass. He says that a vector measure $T$ is a transport path between $\mu^{+}$and $\mu^{-}$if there are sequences of atomic measures $a_{i}$ and $b_{i}$, and paths $G_{i}$ connecting $a_{i}$ to $b_{i}$ such that $a_{i}$ and $b_{i}$ converge weakly to $\mu^{+}$and $\mu^{-}$and $G_{i} \rightarrow T$ weakly in the sense of vector measures. This implies $\operatorname{div}(T)=\mu^{+}-\mu^{-}$in the distribution sense. The energy of any such path is defined by relaxation as

$$
M^{\alpha}(T):=\inf \liminf _{i \rightarrow \infty} M^{\alpha}\left(G_{i}\right),
$$

where the infimum is taken over the set of all possible approximating graph sequences $a_{i}, b_{i}, G_{i}$ of $T$.

Xia's existence theorem states the existence of an optimal transport path between any two Radon measures $\mu^{+}$and $\mu^{-}$with the same mass, provided $1-1 / m \leqslant \alpha \leqslant 1$. Another topic addressed in Section 7 of his paper is the existence of a "transport plan" associated with each path. This roughly says which point of $\mu^{+}$goes to which point of $\mu^{-}$.

It is quite desirable to establish an equivalence between the Lagrangian approach (applied here) and the Eulerian one. None of them has the final desirable form and they are therefore complementary. The Eulerian form is a bit abstract since it does not yield the expected description of the solution as a countable graph. The Lagrangian presentation instead does not immediately yield the elegant embedded solution as a vector measure. Let us just give hints of how this equivalence can be proved. Given an Eulerian solution $T$ with $\mu^{+}$a Dirac mass, we know that it can be assigned a sequence of approximating finite graphs $G_{i}$ without loops, that is, trees. With such finite graphs, we can easily associate patterns $\chi_{i}$ by parameterizing the tree from root to leaves by arc length. Then, by our compactness result (Theorem 8.1) and the lower semicontinuity result on pattern energies, it is easily deduced that there exists a limit Lagrangian solution $\chi$ from the Eulerian one, and whose energy is lower than or equal to the energy of $T$.

Conversely, consider a Lagrangian optimal solution $\chi$, which we can always parameterize by length. Using its filtration structure, we can approximate $\chi$ by a sequence of finite trees $G_{i}$ with the root as a source and whose leaves give an atomic measure $b_{i}$. By monotone convergence, the energy of the $G_{i}$ tends to the energy of $\chi$ and by the result of Section $3, b_{i}$ tends to the irrigation measure of $\chi$. In addition, $G_{i}$, viewed as a vector measure, tends to an Eulerian path $T$. By Xia's relaxed definition of energy, we deduce that the Eulerian energy of $T$ is lower than or equal to the energy of $\chi$. Thus both energies are equal and both formulations are equivalent.

Our results are slightly more restrictive in that we impose $\mu^{+}=\delta_{S}$, a Dirac mass. Also, we do not specify when a feasible solution is attainable. The measure structure on the set of paths (fibers) seems, however, to be a valuable tool. Thanks to the integration tools on the filtration they provide, it can be easily proved that trees irrigating sets with positive Lebesgue measure are incompatible with Poiseuille law [4]. In other terms, infinite irrigation trees seem to be impossible from the fluid mechanics viewpoint. 


\section{Acknowledgements}

We thank Bernard Sapoval and Luigi Ambrosio for many conversations and suggestions. Marc Bernot read this paper carefully and made valuable corrections and improvements. Work supported by C.N.R.S. and the Leonardo da Vinci Italian-French bilateral collaboration fund.

\section{Index of main notations in order of appearance}

- $\chi: \Omega \times \mathbb{R}_{+} \rightarrow \mathbb{R}^{N}$ : set of fibers

- $\chi(p, t) \in \mathbb{R}^{N}:$ position of point $p \in \Omega$ at time $t$

- $t \mapsto \chi(p, t)$ : fiber of $p$

- $[p]_{t}$ : equivalence class of $p$ under the equivalence $p \simeq_{t} q$ if $\chi_{p}(s)=\chi_{q}(s)$ for all $s \in[0, t]$

- $\chi$-vessel: class of equivalence at time $t$ under $\simeq_{t}$

- $\mathcal{V}_{t}(\chi):=\Omega / \simeq_{t}$ : set of $\chi$-vessels at time $t$

- $\sigma_{\chi}(p):=\inf \left\{t \in \mathbb{R}_{+} \mid \chi_{p}(s)\right.$ is constant on $[t, \infty[\}$ : absorption (stopping) time of $p$

- $p$ is absorbed at time $t$ if $\sigma_{\chi}(p) \leqslant t$

- absorbed $\chi$-vessel at time $t$ : if $\sigma_{\chi}(p) \leqslant t$ for a.e. $p \in \Omega$

- $A_{t}(\chi)$ : set of absorbed points at time $t$

- $\chi$-flow: non-absorbed $\chi$-vessel (has positive measure in $\Omega$ )

- $\mathcal{F}_{t}(\chi)$ : set of $\chi$-flows at time $t$

- $F_{t}(\chi)$ : union of all $\chi$-flows at time $t$

- $\chi$-flow evolution: $V_{t}$ such that $V_{t} \in F_{t}(\chi)$ for all $t$ and $V_{t}$ decreasing under inclusion

- $D_{I}\left(V_{t}\right)$ : dispersion of a $\chi$-flow evolution on an interval $I$; set of points which leave the $\chi$-flow without going into another $\chi$-flow $W \neq V_{t}$

- $M_{t}(\chi)=\chi(\Omega, t) \backslash A_{t}(\chi)$ : non-absorbed fibers at time $t$

- $S_{t}(\chi)=M_{t}(\chi) \backslash F_{t}(\chi)$ : spread flow, i.e. set of non-absorbed fibers which do not belong to a $\chi$-flow

- non-spread set of fibers: if $\left|S_{t}(\chi)\right|=0$ for every $t$

- irrigation pattern: set of fibers $\chi$ which is measurable

- $\mathbf{P}_{S}(\Omega)$ : set of all irrigation patterns, i.e. of all measurable sets of fibers

- $c_{\chi}(t)$ : cost functional at time $t$

- $I(\chi)$ cost functional of the irrigation pattern

- $\chi_{n} \rightarrow \chi$ : convergence almost everywhere of irrigation patterns

- $i_{\chi}(p)=\chi\left(p, \sigma_{\chi}(p)\right)$ : irrigation function

- $\mu_{\chi}(A)=\left|i_{\chi}^{-1}(A)\right|$ : irrigation measure induced by $\chi$

- histogram: irrigation pattern $\chi:[0,1] \times \mathbb{R}_{+} \rightarrow \mathbb{R}^{N}$ such that all $\chi$-flows $V \in \mathcal{F}_{t}(\chi)$ are intervals

\section{REFERENCES}

1. Ambrosio, L. Lecture Notes on Optimal Transport Problems. Lecture Notes in Math. 1812, Springer, Heidelberg (2003), 1-52.

2. Ambrosio, L., Fusco, N., \& Pallara, D. Functions of Bounded Variation and Free Discontinuity Problems. Clarendon Press, Oxford (2000). Zbl 0957.49001 MR 96c:35114

3. Bejan, A. \& Errera, M. R. Deterministic tree networks for fluid flow: geometry for minimal flow resistance between a volume and one point. Fractals 5 (1997), 685-695. Zbl 0968.76603 
4. Bernot, M., Caselles, V., \& Morel, J.-M. Physical irrigation. In preparation.

5. Caselles, V. \& Morel, J.-M. Irrigation. Proc. Internat. Workshop on Variational Methods for Discontinuous Structures, with Applications to Image Segmentation and Continuum Mechanics, G. Dal Maso and F. Tomarelli (eds.) (2001).

6. Devillanova, G. \& Solimini, S. Forthcoming.

7. Ekeland, I. \& Temam, R. Convex Analysis and Variational Problems. North-Holland-Elsevier (1976). Zbl 0322.90046

8. Evans, L. C. Partial Differential Equations and Monge-Kantorovich Mass Transfer. Current Developments in Mathematics, Int. Press, Boston, MA (1999). Zbl 0954.35011 MR 2000e:49001

9. Evans, L. C. \& Gangbo, W. Differential equations methods for the Monge-Kantorovich mass transfer problem. Mem. Amer. Math. Soc. 137 (1999). Zbl 0920.49004 MR 99g:35132

10. Evans, L. C. \& GARIEPY, R. F. Measure Theory and Fine Properties of Functions. CRC Press (1992). Zbl 0804.28001 MR 93f:28001

11. Gangbo, W. \& MCCAnn, R. J. The geometry of optimal transportation, Acta Math. 177 (1996), 113161. Zbl 0887.49017 MR 98e:49102

12. Gurtin, M. E. An Introduction to Continuum Mechanics. Academic Press, San Diego (1981). Zbl 0559.73001 MR 84c:73001

13. Halmos, P. R. Measure Theory. Springer, Berlin (1974). Zbl 0283.28001

14. Horton, R. E. Erosional development of streams and their drainage basins: hydrophysical approach to quantitative morphology. Bull. Geol. Soc. Amer. 56 (1945), 275-370.

15. Kantorovich, L. On the transfer of masses. Dokl. Akad. Nauk SSSR 37 (1942), 7-8.

16. Monge, G. Mémoire sur la théorie des déblais et de remblais. Histoire Acad. Roy. Sci. Paris (1781), 666-704.

17. Neveu, J. Bases Mathématiques du Calcul des Probabilités. Masson (1964). Zbl 0203.4990 MR $42 \# 6885$

18. Newman, W. I., Turcotte, D. L., \& Gabrielov, A. M. Fractal trees with side branching. Fractals 5 (1997), 603-614. Zbl 0907.28005

19. Sapoval, B. Universalités et Fractales. Champs 466, Flammarion, Paris (1997). Zbl 1006.00004

20. Strahler, A. N. Quantitative analysis in watershed geomorphology. Trans. Amer. Geophys. Un. 38 (1957), 913-920.

21. Sudakov, V. N. Geometric problems in the theory of infinite dimensional distributions. Proc. Steklov Inst. Math. 141 (1979), 1-178. Zbl 0409.60005 MR 80e:60052

22. Tokunaga, E. Consideration on the composition of drainage networks and their evolution. Geographical Rep. Tokyo Metro. Univ. 13 (1978).

23. Truesdell, C. \& Rajagopal, K. R. An Introduction to the Mechanics of Fluids. Birkhäuser, Boston (2000). Zbl 0942.76001 MR 2000k:76003

24. Villani, C. Topics in Optimal Transportation. Grad. Stud. Math. 58, Amer. Math. Soc., Providence (2003). Zbl pre0190949 MR 1964483

25. West, G. B. The origin of universal scaling laws in biology, Phys. A 263 (1999), 104-113.

26. West, G. B., Brown, J. H., \& EnQuist, B. J. A general model for the structure and allometry of plant vascular systems. Nature 400 (1999), 664-667.

27. West, G. B., Brown, J. H., \& Enquist, B. J. A general model for the origin of allometric scaling laws in biology. Science 276 (1997), no. 4, 122-126.

28. West, G. B., Brown, J. H., \& Enquist, B. J. The fractal dimension of plants. Preprint (2001).

29. XIA, Q. L. Optimal paths related to transport problems. Commun. Contemp. Math. 5 (2003), 251-279. MR 1966259 